\title{
An Earth Pole-Sitter Using Hybrid Propulsion
}

\author{
Matteo Ceriotti ${ }^{1}$ and Colin R. McInnes ${ }^{2}$ \\ University of Strathclyde, Glasgow G1 1XJ, United Kingdom
}

In this paper we investigate optimal pole-sitter orbits using hybrid solar sail and solar electric propulsion (SEP). A pole-sitter is a spacecraft that is constantly above one of the Earth's poles, by means of a continuous thrust. Optimal orbits, that minimize propellant mass consumption, are found both through a shape-based approach, and solving an optimal control problem, using a direct method based on pseudo-spectral techniques. Both the pure SEP case and the hybrid case are investigated and compared. It is found that the hybrid spacecraft allows consistent savings on propellant mass fraction. Finally, is it shown that for sufficiently long missions (more than 8 years), a hybrid spacecraft, based on mid-term technology, enables a consistent reduction in the launch mass for a given payload, with respect to a pure SEP spacecraft.

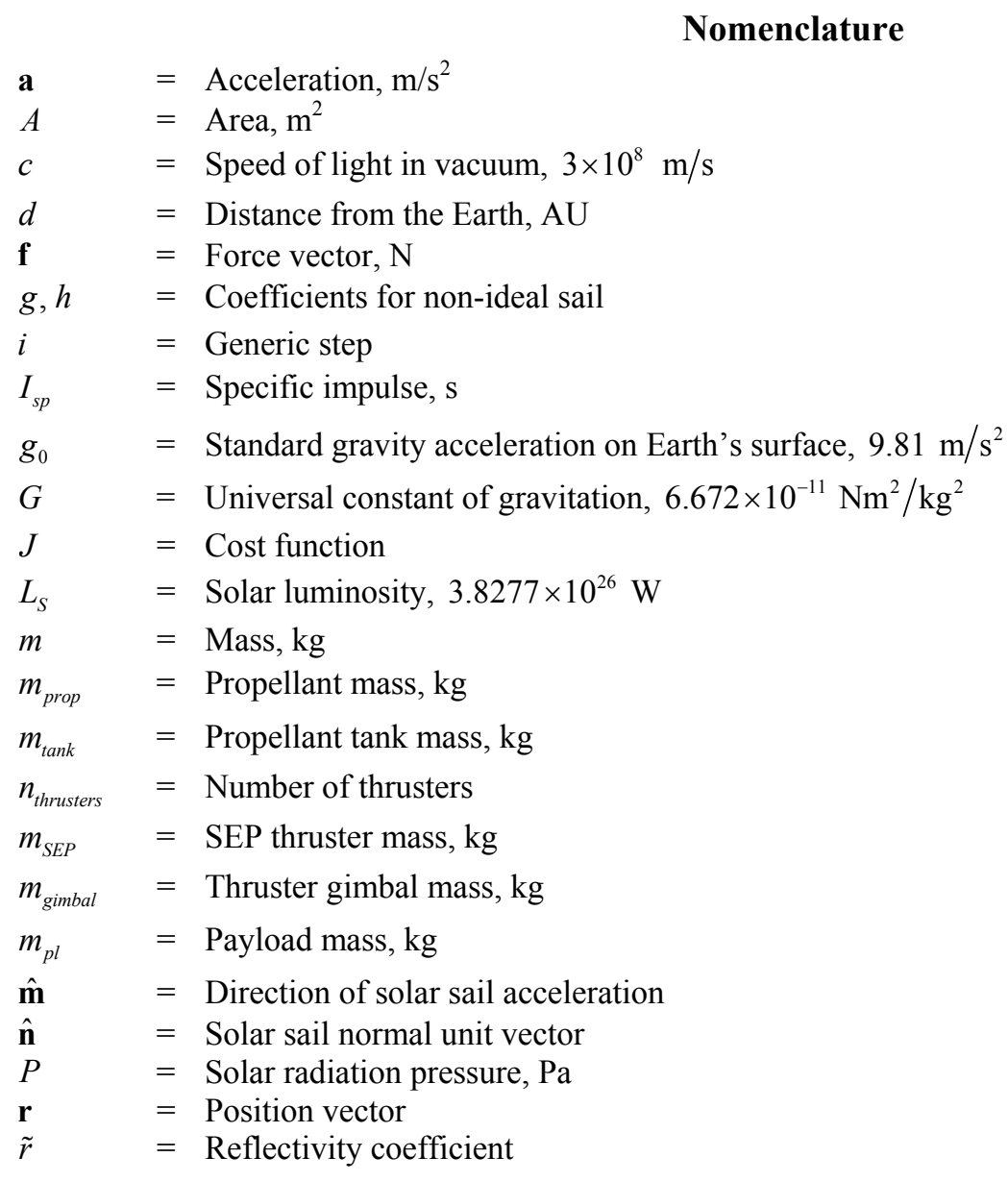

${ }^{1}$ Research Fellow, Advanced Space Concepts Laboratory, Department of Mechanical Engineering, James Weir Building, 75 Montrose Street, matteo.ceriotti@strath.ac.uk, AIAA Member.

${ }^{2}$ Director, Advanced Space Concepts Laboratory, Department of Mechanical Engineering, James Weir Building, 75 Montrose Street, colin.mcinnes@strath.ac.uk, AIAA Member. 


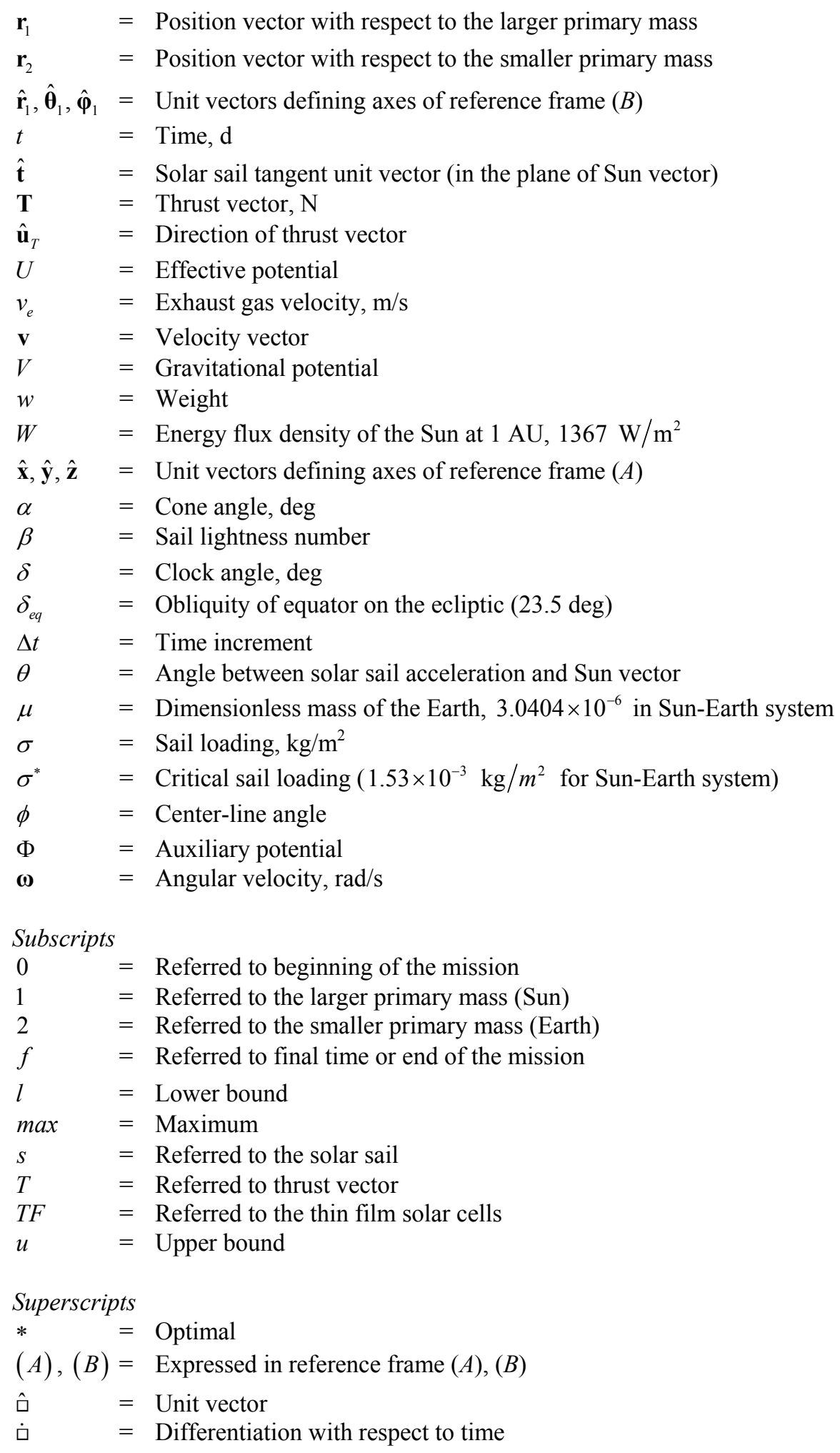

\section{Introduction}

SOLAR electric propulsion (SEP) is a mature technology that provides a spacecraft with a relatively low thrust (of the order of a fraction of a Newton). ${ }^{1}$ However, by accelerating ions to high velocity using an electromagnetic field, its specific impulse is higher (up to several thousands of seconds) than any propulsion system based 
on chemical combustion. Nevertheless, despite the high efficiency, the thrusting time and hence the mission duration is always limited by the amount of propellant on-board.

In contrast, solar sailing (see Ref. 2, pp. 1-29) is a propellant-less spacecraft propulsion system: it exploits the solar radiation pressure due to the solar photons impinging on a large, highly reflecting surface (the sail) to generate thrust. Despite the original idea of solar sailing being rather old, ${ }^{3}$ so far no spacecraft exploiting this intriguing idea has been flown, mainly due to the difficulties of deploying a large, light surface in orbit. However, the concept has never been abandoned; several studies are still ongoing, ${ }^{4-5}$ including mission design, ${ }^{6}$ due to the interesting potential of enabling missions that are not constrained by propellant mass availability. For these reasons, solar sails seem to be suitable for potentially long duration missions that need a small, but continuous, amount of thrust. One example of these applications are interplanetary transfers, ${ }^{7}$ in which a Sun-centered trajectory is generated spiraling inwards or outwards from the Earth's orbit by using a small thrust force. Solar sails have also been investigated to generate artificial equilibrium points, for example in the proximity of the Lagrange points of the Sun-Earth ${ }^{8}$ or Sun-Moon ${ }^{9}$ system: the effect of the sail is that of creating regions in which the spacecraft can be stationary with respect to the two main bodies. ${ }^{10}$ These regions can be consistently far from the classical Lagrange points, thus enabling a whole range of new applications, from telecommunications and data relay to Earth and Moon observation.

Finally, a novel concept is highly non-Keplerian orbits (NKO), in which a continuous acceleration is used to generate periodic orbits that do not follow the natural dynamics of the system. ${ }^{11}$ Examples of these orbits in the twobody problem are displaced Sun-centered orbits for observing the solar poles, or displaced geostationary orbits with a 1-day period, for increasing the capacity of the geostationary ring. ${ }^{12}$ If a second primary is considered, then displaced orbits in the proximity of the Lagrange points are possible. ${ }^{13-15}$

It has already been noted that at present the technology readiness level (TRL) of a spacecraft propelled with a solar sail is relatively low, making such a mission high risk. Another disadvantage of solar sailing is that it is not possible to generate an acceleration component directed towards the Sun. This intrinsic limitation prevents the sail being used for a number of applications.

Considering advantages and limitations of both SEP and solar sailing, the idea of a hybrid propulsion spacecraft, combining a solar sail and SEP arises. Hybridizing the two propulsion systems is a recent idea, ${ }^{16}$ nevertheless research is flourishing in this field, investigating its potential for novel, interesting applications. Baig and McInnes proposed the use of a hybrid sail for generating artificial equilibria above $L_{1}$ in the Sun-Earth system for Earth observation; ${ }^{17}$ Mengali and Quarta investigated optimal interplanetary transfers to Venus and Mars using an indirect optimization method; ${ }^{18-19}$ Quarta et al. also considered hybridizing SEP with an electric sail ${ }^{20}$ Simo and McInnes exploited hybrid propulsion to find displaced periodic orbits in the Earth-Moon system; ${ }^{21}$ finally, JAXA has developed a powered hybrid solar sail demonstrator, IKAROS. ${ }^{22}$

The reason for this interest is due to the fact that in the hybrid system, at the cost of increased spacecraft complexity, the two propulsion systems complement each other, cancelling their reciprocal disadvantages and limitations. In principle, SEP can provide thrust in any direction (through attitude maneuvers or a gimbal-mounted thruster), thus it can provide the missing acceleration component towards the Sun, that the sail cannot generate. Similarly, the hybrid spacecraft can be seen as an SEP spacecraft, in which an auxiliary solar sail provides part of the acceleration, enabling saving of propellant, and lower demand on the electric thruster, possibly with some intervals in which it could be turned off. Moreover, the reliability of the whole system is increased, for two reasons. The first is that the solar sail is only providing part of the necessary acceleration, and hence it can be smaller than what would be required for a pure sail spacecraft. The second is that the spacecraft is not relying on the sail only; if, for example, the sail fails to deploy, then the SEP is still available: in this case, a degraded, possibly shorter mission can be foreseen. In this sense, the hybrid spacecraft can be seen as a way to gradually introduce solar sails for space applications, and hence to reduce the advancement degree of difficulty $\left(\mathrm{AD}^{2}\right.$, Ref. 23$)$ in the TRL scale.

In this paper, we present the orbital dynamics of a hybrid propulsion spacecraft, applied to a particular family of NKO: so-called pole-sitter orbits. A pole-sitter is a spacecraft that is constantly above one of the Earth's poles, i.e. lying on the Earth's polar axis. ${ }^{24}$ The distance from the pole can vary during the mission or be constant. In the latter case the spacecraft is stationary in an Earth-centered, Earth-fixed reference frame, and the problem can be formulated as finding the required acceleration for maintaining an artificial equilibrium point. If the gravitational attraction of the Sun is also considered and a Sun-Earth rotating frame is considered, then the pole-sitter spacecraft describes, in the Sun-Earth frame, a non-Keplerian orbit, as will be shown in the following sections.

The concept of having a spacecraft in a position that allows direct-link telecommunication and visibility of one of the Earth poles has been already investigated. ${ }^{24-26}$ The pole-sitter can provide a platform for continuous, real-time, medium-resolution observation of the Earth poles, with a full hemispheric view. 
Traditionally, continuous Earth observation is performed with equatorial geostationary platforms, which cannot cover high latitude regions (see for example Ref. 27, pp. 165-168). For polar areas, a constellation of spacecraft with highly-inclined low or medium Earth orbits could also be used: ${ }^{28}$ in this case, the observation is not continuous, but it relies on the passage of one spacecraft above the point of interest. The frequency of the passages depends on the number of spacecraft in the constellation and the configuration of the constellation. In any case, more than one spacecraft is required, and the full hemispheric view must be reconstructed through a set of different images.

A continuous, hemispherical, real-time view of the pole will enable a wide range of new applications. It is well known that line-of-sight telecommunications to conventional spacecraft in geostationary orbits is not possible at high latitudes and polar regions. Telecommunication with polar regions will be a key issue in future as changes to the arctic ice pack opens navigation channels for shipping. Also, the pole-sitter spacecraft could provide a platform for real-time observation of the poles for climate science at modest resolution, as opposed to periodic images that can be obtained by classical high-inclination orbits.

A stationary spacecraft in the rotating Sun-Earth system for Earth observation and data relaying was investigated using a pure solar sail by McInnes in Ref. 25. It was found that it is not possible to have a pure sail permanently fixed above a pole of the Earth. In addition, if a realistic sail is used, the volume in which equilibria are possible on the night side of the Earth (far from the Sun) is limited: high sail performances are required to maintain a pure sail in these regions, where the spacecraft can guarantee coverage of the North Pole in winter or the south pole in summer.

This work was successively extended by Baig and McInnes: ${ }^{17}$ in that paper, it was proposed to place a spacecraft in the same artificial equilibrium points of the Sun-Earth rotating system, but using hybrid SEP/sail propulsion. The authors showed that, considering an SEP spacecraft with a given level of thrust, the regions in which the spacecraft can stay in equilibrium are extended by adding a solar sail. Furthermore, for the same level of thrust, a sail allows the spacecraft to stay closer to the pole, or to stay in the same point with a lower level of thrust, thus saving propellant. Nevertheless, a stationary spacecraft in the Sun-Earth rotating frame does not follow the apparent rotation of the Earth's polar axis. Therefore, the full hemispheric view of the pole only happens at one particular time in the year, while at other epochs the polar region is partially out of sight.

In this work, we propose an extension of both the pure-SEP pole-sitter proposed by Driver, ${ }^{24}$ and the hybrid stationary spacecraft proposed by Baig and McInnes. ${ }^{17}$ With respect to the former, this work extends Driver's analysis in two directions: the first is considering a hybrid propulsion spacecraft. The second is the use of optimal control theory to find optimal orbits (with respect to SEP fuel consumption). This gives more flexibility for trading off the shape of the orbit (namely the excursion along the polar axis), taking into account constraints on the altitude, while minimizing propellant consumption. This will result in optimal orbits that can produce an additional saving in propellant mass. In addition, it will be shown that the addition of the solar sail helps both by saving propellant, and, in some cases, in generating periods in which the SEP can be switched off.

In the latter work, ${ }^{17}$ Baig and McInnes introduced the hybrid propulsion spacecraft, but the analysis was performed only at two artificial equilibrium points, which are above the Earth pole at summer and winter solstices respectively. The continuous pole-sitter orbits presented in this paper using hybrid SEP/solar sail propulsion are a practical realization of the solar sail pole-sitter orbits proposed earlier by Forward. ${ }^{26}$

The paper is organized as follows. In section II, the equations of motion of the hybrid SEP/sail spacecraft will be presented. Section III presents the orbital dynamics of a pole-sitter spacecraft, and will introduce a shape-based approach to find the control law for enabling a given pole-sitter orbit. However, this method does not lead to fully optimal solutions, for which an optimal control problem is to be solved. In this work, a direct method based on pseudo-spectral techniques will be used. Section IV will present, after a brief summary of the technique, the optimal orbits that were designed. Finally, Section V compares, with a preliminary mass budget, the launch mass of the SEP spacecraft and the hybrid spacecraft in different scenarios.

\section{Equations of Motion}

The circular restricted three-body problem (CR3BP) framework is considered. This model describes the motion of an infinitesimal mass (the spacecraft) under the gravitational attraction of two large masses (primaries), which are orbiting around their common center of mass in circular motion. The model neglects the influence of the spacecraft on the motion of the primaries. As is common, a rotating reference frame is considered, in which the origin is in the center of mass of the system, the $\hat{\mathbf{x}}$-axis is collinear with the two primaries, pointing towards the smaller mass, the $\hat{\mathbf{z}}$-axis is aligned with the angular velocity of the system, and the $\hat{\mathbf{y}}$-axis completes the right-handed Cartesian reference frame (see Fig. 1). This reference frame will be referred to as $(A)$ :

4

American Institute of Aeronautics and Astronautics 


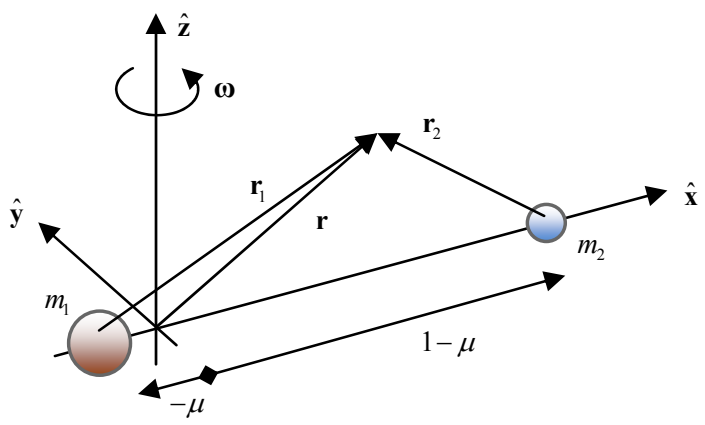

Fig. 1. Reference frame $(A)$ : the restricted three body problem.

$$
(A):\left\{\begin{array}{l}
\hat{\mathbf{x}} \\
\hat{\mathbf{y}} \\
\hat{\mathbf{z}}
\end{array}\right.
$$

Note that this frame is not inertial. In the following, all the vectors are expressed in this frame, unless specified by a different superscript. Therefore, the superscript $(A)$ will be omitted when not strictly needed.

The masses of the two primaries are denoted $m_{1}$ and $m_{2}$ (with $m_{1}>m_{2}$ ), and $\boldsymbol{\omega}=\omega \hat{\mathbf{z}}$ the angular velocity of the system. The equations that describe the motion of the spacecraft in this system are:

$$
\ddot{\mathbf{r}}+2 \boldsymbol{\omega} \times \dot{\mathbf{r}}+\boldsymbol{\omega} \times(\boldsymbol{\omega} \times \mathbf{r})=-\nabla V+\mathbf{a}
$$

where $\mathbf{r}$ is the position vector, $\mathbf{a}$ is the acceleration due to external, non-gravitational forces (i.e. thrust), and $V$ is the gravitational potential due to the primaries:

$$
V(\mathbf{r})=-\left(\frac{1-\mu}{r_{1}}+\frac{\mu}{r_{2}}\right)
$$

The non-dimensional mass of the smaller primary $\mu$ is defined as:

$$
\mu=m_{2} /\left(m_{1}+m_{2}\right)
$$

such that the gravitational constants of the two primaries become:

$$
\begin{aligned}
G m_{1} & =1-\mu \\
G m_{2} & =\mu
\end{aligned}
$$

where $G$ is the universal constant of gravitation.

In the following, the system of equations will be used in their canonical non-dimensional form, that is assuming $\omega=1$ and the unit of distance being the separation of the two primaries. With these assumption, the position along the $\hat{\mathbf{x}}$-axis of $m_{1}$ is $-\mu$, and the position of $m_{2}$ is $1-\mu$. In this work, the primary $m_{1}$ is the Sun, and the primary $m_{2}$ is the Earth: for these bodies, $\mu=3.0404 \cdot 10^{-6}$. The two vectors $\mathbf{r}_{1}$ and $\mathbf{r}_{2}$ are the position of the spacecraft with respect to the two primaries $m_{1}$ and $m_{2}$ respectively. These are defined as (see Fig. 1):

If an auxiliary potential is now defined as:

$$
\begin{aligned}
& \mathbf{r}_{1}=\mathbf{r}+\left[\begin{array}{lll}
0 & 0 & \mu
\end{array}\right]^{T} \\
& \mathbf{r}_{2}=\mathbf{r}-\left[\begin{array}{lll}
0 & 0 & 1-\mu
\end{array}\right]^{T}
\end{aligned}
$$

it can be shown that

$$
\Phi(\mathbf{r})=-\frac{1}{2}\left(x^{2}+y^{2}\right)
$$

$$
\boldsymbol{\omega} \times(\boldsymbol{\omega} \times \mathbf{r})=\nabla \Phi
$$

and hence the effective potential can be introduced as

allowing Eq. (1) to be written as:

$$
U=V+\Phi
$$




$$
\ddot{\mathbf{r}}+2 \boldsymbol{\omega} \times \dot{\mathbf{r}}=-\nabla U+\mathbf{a}
$$

In this work, the acceleration $\mathbf{a}$ is from two contributions: one due to the solar radiation pressure on the spacecraft sail, $\mathbf{a}_{s}$; the second is provided by the thrust of the solar electric propulsion (SEP) system, $\mathbf{a}_{T}$. The two fractions of the total acceleration vector will be described in the following sub-sections.

\section{A. Solar Sailing}

Solar sailing is the exploitation of the solar radiation pressure produced by photons on a surface of the spacecraft (the sail) to produce a force, and thus an acceleration, without using any propellant. In this section a non-perfect solar sail force model will be presented that will be used in the following analysis.

According to Ref. 2, p. 49, an approximate expression for the force on a flat surface, of area $A$, whose reflection coefficient is $\tilde{r}$, is:

$$
\mathbf{f}_{s}=P A\left[(1+\tilde{r}) \cos ^{2} \alpha \hat{\mathbf{n}}+(1-\tilde{r}) \cos \alpha \sin \alpha \hat{\mathbf{t}}\right]
$$

where $P$ is the solar radiation pressure at distance $r_{1}$ from the Sun (approximated as a point source), expressed as:

$$
P=\frac{1}{c} \frac{L_{S}}{4 \pi r_{1}^{2}}
$$

with $L_{S}$ being the solar luminosity and $c$ the speed of light. The term $L_{S} / 4 \pi r_{1}^{2}$ is the energy flux of the Sun at distance $r_{1}$. The force is expressed through its two components: one normal to the sail, along $\hat{\mathbf{n}}$, and one perpendicular to the sail, in the plane containing the Sun vector $\hat{\mathbf{r}}_{1}$, along $\hat{\mathbf{t}}$ (see Fig. 2).

The angle between the direction of the Sun vector $\hat{\mathbf{r}}_{1}$ and the normal to the solar sail $\hat{\mathbf{n}}$ is known as the cone angle $\alpha$. It holds that:

$$
\cos \alpha=\hat{\mathbf{n}} \cdot \hat{\mathbf{r}}_{1}
$$

Equation (4) assumes specular reflection and absorption of the photons. Scattered reflection and emission by reradiation are neglected. The spacecraft has a relatively large surface, the solar sail, which provides the necessary acceleration. Ideally a solar sail is made of a light, thin film of highly reflective material, since maximizing its reflectivity maximizes the magnitude of the force, as can be seen from Eq. (4) when $\tilde{r} \rightarrow 1$.

In the case under consideration, we cannot assume the sail material to be uniform. In fact, part of the sail is covered with thin film solar cells (TFSC), that provide the necessary electrical power for the SEP engine. The TFSC has different reflection properties than the rest of the sail (its reflectivity is lower, as part of the light is absorbed and converted into solar power).

An area $A_{s}$ of the sail is covered with highly reflective material, with reflectivity coefficient $\tilde{r}_{s}$, while the remaining surface $A_{T F}$ is covered with TFSC with reflectivity coefficient $\tilde{r}_{T F}$. The total force then becomes:

$$
\begin{aligned}
\mathbf{f}_{s} & =P A_{s}\left[\left(1+\tilde{r}_{s}\right) \cos ^{2} \alpha \hat{\mathbf{n}}+\left(1-\tilde{r}_{s}\right) \cos \alpha \sin \alpha \hat{\mathbf{t}}\right]+P A_{T F}\left[\left(1+\tilde{r}_{T F}\right) \cos ^{2} \alpha \hat{\mathbf{n}}+\left(1-\tilde{r}_{T F}\right) \cos \alpha \sin \alpha \hat{\mathbf{t}}\right]= \\
& =P\left[\left(A_{T F}\left(1+\tilde{r}_{T F}\right)+A_{s}\left(1+\tilde{r}_{s}\right)\right) \cos ^{2} \alpha \hat{\mathbf{n}}+\left(A_{T F}\left(1-\tilde{r}_{T F}\right)+A_{s}\left(1-\tilde{r}_{s}\right)\right) \cos \alpha \sin \alpha \hat{\mathbf{t}}\right]
\end{aligned}
$$

Considering that the total sail area is $A=A_{T F}+A_{s}$ :

$$
\mathbf{f}_{s}=P A\left[\left(1+\tilde{r}_{s}+\frac{A_{T F}}{A}\left(\tilde{r}_{T F}-\tilde{r}_{s}\right)\right) \cos ^{2} \alpha \hat{\mathbf{n}}+\left(1-\tilde{r}_{s}-\frac{A_{T F}}{A}\left(\tilde{r}_{T F}-\tilde{r}_{s}\right)\right) \cos \alpha \sin \alpha \hat{\mathbf{t}}\right]
$$

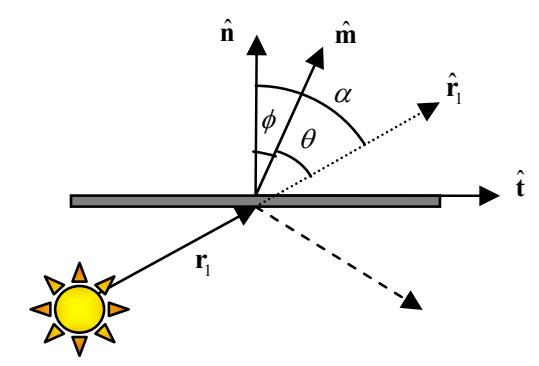

Fig. 2. Definition of the cone and center-line angles (plane of the figure is perpendicular to the sail, containing the Sun vector $\left.r_{1}\right)$. 
Defining the two coefficients, functions of the two material reflectivities and area ratio:

$$
\begin{aligned}
& g=1+\tilde{r}_{s}+\frac{A_{T F}}{A}\left(\tilde{r}_{T F}-\tilde{r}_{s}\right) \\
& h=1-\tilde{r}_{s}-\frac{A_{T F}}{A}\left(\tilde{r}_{T F}-\tilde{r}_{s}\right)
\end{aligned}
$$

the force on the sail becomes:

$$
\mathbf{f}_{s}=P A[g \cos \alpha \hat{\mathbf{n}}+h \sin \alpha \hat{\mathbf{t}}] \cos \alpha
$$

The acceleration of the spacecraft (of mass $m$ ) due to this force can be expressed completely inserting Eq. (5) into (7), and dividing by the spacecraft mass $m$ :

$$
\mathbf{a}_{s}=\frac{\mathbf{f}_{s}}{m}=\frac{1}{c} \frac{L_{S}}{4 \pi r_{1}^{2} m} A[g \cos \alpha \hat{\mathbf{n}}+h \sin \alpha \hat{\mathbf{t}}] \cos \alpha
$$

The system loading $\sigma=m / A$ is now defined as the ratio between the mass of the spacecraft and the total solar sail area, and the dimensionless parameter $\beta$, commonly known as sail lightness number, is defined as

$$
\beta=\frac{\sigma^{*}}{\sigma}
$$

where the critical sail loading $\sigma^{*}$ is a constant for the Sun-Earth system:

$$
\sigma^{*}=\frac{2}{c} \frac{L_{S}}{4 \pi} \frac{1}{1-\mu} \cong 1.53 \cdot 10^{-3} \mathrm{~kg} / \mathrm{m}^{2}
$$

Here $1-\mu=G m_{1}$ is the dimensional gravitational parameter of the Sun: hence, the sail lightness number can also be interpreted as the ratio between the gravitational acceleration of the Sun and the solar sail acceleration.

By substituting Eqs. (9) and (10) in (8), a more compact form is obtained as:

$$
\mathbf{a}_{s}=\frac{1}{2} \beta \frac{1-\mu}{r_{1}^{2}}[g \cos \alpha \hat{\mathbf{n}}+h \sin \alpha \hat{\mathbf{t}}] \cos \alpha
$$

Since the mass of the spacecraft varies due to propellant consumption, the parameter $\beta$ varies during the mission. Therefore, the (constant) lightness number at beginning of life $\beta_{0}$, is defined such that $\beta=\beta_{0} \frac{m_{0}}{m}$, where $m_{0}$ is the mass of the spacecraft at a initial reference time $t_{0}$, and therefore the solar sail acceleration becomes an explicit function of the mass:

$$
\mathbf{a}_{s}=\frac{1}{2} \beta_{0} \frac{m_{0}}{m} \frac{1-\mu}{r_{1}^{2}}[g \cos \alpha \hat{\mathbf{n}}+h \sin \alpha \hat{\mathbf{t}}] \cos \alpha
$$

If the unit vector $\hat{\mathbf{m}}$ is defined in the direction of $\mathbf{a}_{s}$, Eq. (12) can be rewritten as

$$
\mathbf{a}_{s}=a_{s} \hat{\mathbf{m}}=\frac{1}{2} \beta_{0} \frac{m_{0}}{m} \frac{1-\mu}{r_{1}^{2}} \sqrt{g^{2} \cos ^{2} \alpha+h^{2} \sin ^{2} \alpha} \cos \alpha \hat{\mathbf{m}}
$$

Therefore, the solar sail acceleration depends on the mass of the spacecraft, on its position with respect to the Sun $\mathbf{r}_{1}$, and on its attitude, through $\alpha$ and $\hat{\mathbf{m}}$, in addition to other technological and physical parameters. The direction of the solar sail acceleration $\hat{\mathbf{m}}$ with respect to the Sun-line is identified by the angle $\theta$ (see Fig. 2). It is possible to find a relationship between $\theta$ and $\alpha$, such that the acceleration (13) can be expressed as a function of the cone angle $\alpha$ only. This is achieved by defining the center-line angle $\phi$, as the angle between $\hat{\mathbf{m}}$ and $\hat{\mathbf{n}}$. Decomposing the acceleration (12) into its two components, and taking their ratio, it can be seen that:

$$
\tan \phi=\frac{a_{s, t}}{a_{s, n}}=\frac{h}{g} \tan \alpha
$$

Then, considering the geometric relationship $\phi=\alpha-\theta$ (shown in Fig. 2), and solving for $\theta$, it can be seen that:

$$
\tan \theta=\frac{(g-h) \tan \alpha}{g+h \tan ^{2} \alpha}
$$

Note that the expression for the solar sail acceleration (13) reduces to the ideal case (compare with Ref. 2, p. 216) 


$$
\mathbf{a}_{s}=\beta \frac{1-\mu}{r_{1}^{2}}\left(\hat{\mathbf{n}} \cdot \hat{\mathbf{r}}_{1}\right)^{2} \hat{\mathbf{n}}
$$

if there is no mass consumption ( $m=m_{0}$ ) and a perfectly reflecting sail $\left(A_{T F}=0, \tilde{r}_{s}=1\right.$ ), and the sail force is purely normal to the sail surface. In the case of partially reflecting material for the sail, the sail force is not purely normal to the sail, but instead a tangential component is introduced.

An intrinsic limitation of solar sailing is that the thrust force can only point away from the Sun. This is equivalent to stating that the normal vector $\hat{\mathbf{n}}$ cannot be directed towards the Sun, which translates into the attitude constraint:

$$
0 \leq \alpha \leq \pi / 2
$$

As discussed, the cone angle $\alpha$ defines the pitch attitude of the solar sail with respect to the Sun direction $\hat{\mathbf{r}}_{1}$. The attitude of the sail around this vector is determined through the so called clock angle $\delta$ : this is the angle, measured around $\hat{\mathbf{r}}_{1}$, of the component of $\hat{\mathbf{n}}$ perpendicular to $\hat{\mathbf{r}}_{1}$, starting from the vertical plane (see Fig. 3). To uniquely define the direction of $\hat{\mathbf{m}}$ in space, it is useful to define a new reference frame, named $(B)$, depending on the position of the spacecraft, defined as:

$$
(B):\left\{\begin{array}{l}
\hat{\mathbf{r}}_{1} \\
\hat{\boldsymbol{\theta}}_{1} \equiv \frac{\hat{\mathbf{z}} \times \hat{\mathbf{r}}_{1}}{\left|\hat{\mathbf{z}} \times \hat{\mathbf{r}}_{1}\right|} \\
\hat{\boldsymbol{\varphi}}_{1} \equiv \hat{\mathbf{r}}_{1} \times \hat{\boldsymbol{\theta}}_{1}
\end{array}\right.
$$

Note that this reference frame coincides with a spherical reference frame (radius, right ascension, declination) centered on the Sun.

A vector defined in the reference frame $(B)$ can be expressed in frame $(A)$ considering the transformation:

$$
\mathbf{u}^{(A)}=\left[\begin{array}{lll}
\hat{\mathbf{r}}_{1} & \hat{\boldsymbol{\theta}}_{1} & \hat{\boldsymbol{\varphi}}_{1}
\end{array}\right]^{T} \mathbf{u}^{(B)}
$$

Also note that the clock angles of $\hat{\mathbf{n}}$ and $\hat{\mathbf{m}}$ are coincident, therefore the same symbol $\delta$ will be used.

The Cartesian coordinates of the unit vectors $\hat{\mathbf{n}}$ and $\hat{\mathbf{m}}$, expressed as a function of cone and clock angles, can be defined in frame $(B)$ by:

$$
\hat{\mathbf{n}}^{(B)}=\left[\begin{array}{c}
\cos \alpha \\
\sin \alpha \sin \delta \\
\sin \alpha \cos \delta
\end{array}\right] ; \hat{\mathbf{m}}^{(B)}=\left[\begin{array}{c}
\cos \theta \\
\sin \theta \sin \delta \\
\sin \theta \cos \delta
\end{array}\right]
$$

In this paper, we assume a reflectivity $\tilde{r}_{s}=0.9, \tilde{r}_{T F}=0.4$ for the sail and the TFSC respectively. ${ }^{16}$ It is also assumed that the TFSC occupies $5 \%$ of the total area of the sail, hence $A_{T F} / A=0.05$. This is estimated to be a conservative value, based on previous studies. ${ }^{17}$ The actual area of the thin film solar cells can be determined once the requirements on the thrust are defined, by sizing the power subsystem.

The analysis will be performed for a set of values of the lightness number $\beta_{0}$, ranging from 0 (pure SEP) to 0.1 , that represents a future estimation of sail performance. A value of 0.05 can be assumed for a near-term system. ${ }^{29}$

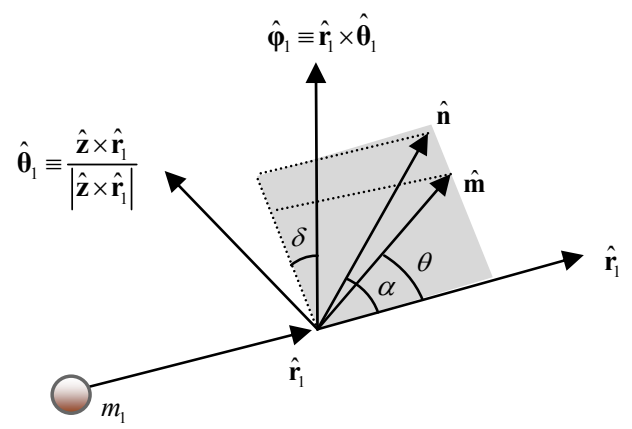

Fig. 3. Frame $(B)$ and solar sail cone and clock angles. 


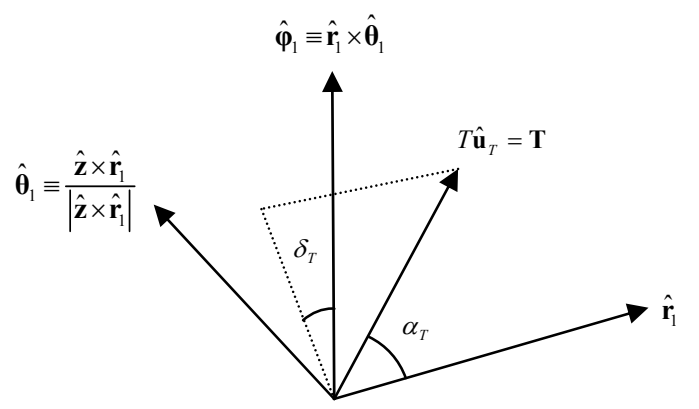

Fig. 4. Thrust cone and clock angles.

\section{B. SEP Thruster}

The thruster is assumed to be steerable and can provide an adjustable thrust force up to a maximum value $T_{u}$. The direction of the thrust vector $\mathbf{T}$ can be defined through the unit vector $\hat{\mathbf{u}}_{T}$, i.e. $\mathbf{T}=T \hat{\mathbf{u}}_{T}$. The relation between the thrust and the resulting spacecraft acceleration is therefore:

$$
\mathbf{a}_{T}=a_{T} \hat{\mathbf{u}}_{T}=\frac{T}{m} \hat{\mathbf{u}}_{T}
$$

Analogously to $\hat{\mathbf{n}}$, the unit vector $\hat{\mathbf{u}}_{T}$ can also be expressed in terms of cone and clock angles $\alpha_{T}, \delta_{T}$. These angles are defined in the same way as for the solar sail (Fig. 4). Therefore, in frame (B):

$$
\hat{\mathbf{u}}_{T}^{(B)}=\left[\begin{array}{c}
\cos \alpha_{T} \\
\sin \alpha_{T} \sin \delta_{T} \\
\sin \alpha_{T} \cos \delta_{T}
\end{array}\right]
$$

In the case of the solar sail, the parameterization through cone and clock angle is useful due to the relation of the cone angle to the magnitude of the force. In the case of the SEP thrust, the cone and clock angles have no particular meaning: the choice is dictated by having the same parameterization for the two parts of the acceleration.

In this work, a specific impulse of $I_{s p}=3000 \mathrm{~s}$ is assumed, based on current ion engine technology (existing NSTAR/DS $1^{30}$ or EADS/Astrium RIT-XT). However, values up to $4500 \mathrm{~s}$ are expected to be achieved in the near future (e.g. BepiColombo's QinetiQ T6). ${ }^{1}$ The value of the maximum thrust $T_{u}$ is set to $0.2 \mathrm{~N}$, for a spacecraft with $1000 \mathrm{~kg}$ of initial mass.

\section{III.Shape-Based Approach}

A pole-sitter spacecraft, during the operations phase of its mission, is constantly aligned with the polar axis of the Earth. If we neglect precession of the equinoxes and nutation (which are long-term, and thus irrelevant in this analysis), the polar axis of the Earth does not change its direction while the Earth is orbiting the Sun. Therefore, in the rotating reference frame $(A)$, the same axis rotates with a motion of apparent precession (Fig. 5). Its angular velocity is the opposite of that of frame $(A)$, or $-\boldsymbol{\omega}$. Therefore the polar axis spans a full conical surface in frame $(A)$ every year. The cone half angle is the tilt of the axis relative to the ecliptic, i.e. $\delta_{e q}=23.5$ deg . Therefore, the pole-sitter shall follow the Earth's polar axis, and describe a 1-year-periodic orbit.

The equations of motion of the hybrid propulsion spacecraft, in the $(A)$ reference frame, can be found starting from (3) and substituting the two accelerations due to the sail (13) and SEP (18):

$$
\ddot{\mathbf{r}}+2 \boldsymbol{\omega} \times \dot{\mathbf{r}}=-\nabla U+a_{s} \hat{\mathbf{m}}^{(A)}+a_{T} \hat{\mathbf{u}}_{T}^{(A)}
$$

The equations of motion (20) require the additional equation that relates the mass consumption with the thrust force. From Newton's law:

$$
T=m a_{T}=\dot{m} v_{e}
$$

where $\dot{m}$ is the propellant mass flow and $v_{e}$ is the velocity of the exhaust gas. This parameter is commonly expressed through the engine specific impulse, viz. $v_{e}=I_{s p} g_{0}$, being $g_{0}=9.81 \mathrm{~m} / \mathrm{s}^{2}$ the gravitational acceleration on the surface of the Earth. 


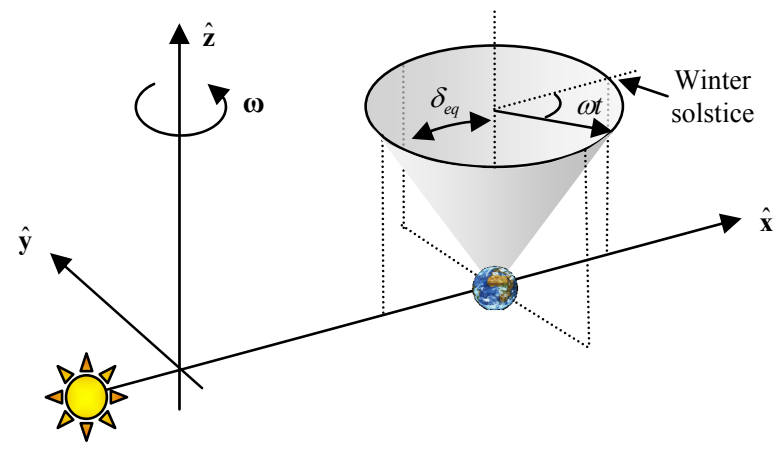

Fig. 5. Apparent precession of the Earth's polar axis due to rotation of reference frame $(A)$. The figure illustrates the different directions of the axis over one year.

It is convenient to rewrite Eqs. (20) and (21) as a first order system by introducing the state vector $\mathbf{x}=\left[\begin{array}{lll}\mathbf{r} & \mathbf{v} & m\end{array}\right]^{T}$, so that:

$$
\dot{\mathbf{x}}=\left[\begin{array}{c}
\dot{\mathbf{r}} \\
\dot{\mathbf{v}} \\
\dot{m}
\end{array}\right]=\left[\begin{array}{c}
\mathbf{v} \\
-\nabla U-2 \boldsymbol{\omega} \times \mathbf{v}+a_{s} \hat{\mathbf{m}}^{(A)}+a_{T} \hat{\mathbf{u}}_{T}^{(A)} \\
-T / v_{e}
\end{array}\right]
$$

The dynamics of the spacecraft (22) is to be constrained, to follow the apparent precession of the polar axis, and hence maintain the pole-sitter condition. It is assumed that the initial time $t_{0}=0$ coincides with the winter solstice, and therefore the pole-sitter spacecraft is on the cone at position:

$$
\mathbf{r}(t)=\left[\begin{array}{c}
d(t) \sin \delta_{e q} \cos \omega t+(1-\mu) \\
-d(t) \sin \delta_{e q} \sin \omega t \\
d(t) \cos \delta_{e q}
\end{array}\right]
$$

where $d(t)$ is the distance from the centre of the Earth, and is in general a continuous function of time. Without loss of generality, due to the symmetry of the problem in the $\hat{\mathbf{x}}-\hat{\mathbf{y}}$ plane, the North Pole case is considered in this work.

Note that in a pure SEP spacecraft, the term $a_{s} \hat{\mathbf{m}}^{(A)}$ in system (22) is null, and thus the coupling between the equation of the mass and the equations for $\mathbf{r}, \mathbf{v}$ is through the acceleration $a_{T}$, that is defined as a function of the control $T$ through Eq. (21). However, as long as the SEP system can provide the necessary thrust (i.e. the thrust is not saturated), then the system (22) can be scaled in mass, without affecting the evolution of the other states. Therefore, the optimal solution in terms of $\mathbf{r}(t), \mathbf{v}(t)$ can be found using a unitary initial mass. The actual evolution of the mass over time for a given spacecraft is found multiplying the mass fraction by the initial mass of the spacecraft. This means that the initial mass is only a scaling factor for the same equation, and does not affect the motion of the spacecraft. Furthermore, if a periodic orbit is considered, and until the propulsion system is able to provide the necessary peak thrust, the propellant mass fraction for each orbit period does not depend on the initial mass. This is not the case for the hybrid spacecraft: in fact, the spacecraft mass is coupled with the equations of motion through the term $a_{s}$, which depends explicitly on the mass, and the system cannot be scaled. In fact, while with SEP the magnitude of the thrust can be varied to compensate for the mass reduction, the magnitude of the thrust generated by the sail cannot be controlled independently from its direction. This means that the acceleration provided by the sail, for a given clock angle, is higher as the mass decreases, and the propellant mass fraction needed for each orbit period is different depending on the actual value of the initial mass in that period. However, for the cases under consideration, it was observed that the propellant mass fraction decreases when the spacecraft mass decreases. Therefore, it is conservative to assume that the propellant mass fraction for the first orbital period is the same for all the other periods. 


\section{A. Thrust Vector Optimization}

An optimal control problem generally requires a first guess that is accurate enough to allow the optimizer to converge quickly and smoothly to a locally optimal solution. In this work, the first guess is generated by using a shape-based approach, ${ }^{31}$ in which a specific orbit for the spacecraft is assigned, and then the controls that enable that orbit are obtained from the equations of motion. That is, once the kinematics of the orbit is assigned through the position $\mathbf{r}(t)$ function of time, find the controls $\mathbf{u}(t)$ at each instant of time that enable that orbit. The total acceleration needed a can be computed from Eq. (3):

$$
\mathbf{a}=\ddot{\mathbf{r}}+2 \boldsymbol{\omega} \times \dot{\mathbf{r}}+\nabla U
$$

In fact, the right hand side of Eq. (24) is fully determined when $\mathbf{r}(t)$ is given. The acceleration can also be expressed in modulus, cone and clock angles in the following way. The modulus is simply:

$$
a=|\ddot{\mathbf{r}}+2 \boldsymbol{\omega} \times \dot{\mathbf{r}}+\nabla U|
$$

The cone and clock angles $\alpha_{a}$ and $\delta_{a}$ can be found rewriting $\hat{\mathbf{a}}$ in frame $(B)$ :

From which it can be seen that:

$$
\left[\begin{array}{c}
\cos \alpha_{a} \\
\sin \alpha_{a} \sin \delta_{a} \\
\sin \alpha_{a} \cos \delta_{a}
\end{array}\right]=\left[\begin{array}{lll}
\hat{\mathbf{r}}_{1} & \hat{\boldsymbol{\theta}}_{1} & \hat{\mathbf{\varphi}}_{1}
\end{array}\right] \hat{\mathbf{a}}=\hat{\mathbf{a}}^{(B)}
$$

$$
\begin{aligned}
& \alpha_{a}=\arccos \hat{a}_{r_{1}}^{(B)} \\
& \delta_{a}=\arctan \left(\frac{\hat{a}_{\theta_{1}}^{(B)}}{\sin \alpha_{a}}, \frac{\hat{a}_{\varphi_{1}}^{(B)}}{\sin \alpha_{a}}\right)
\end{aligned}
$$

in which arctan represents the four-quadrant inverse of the tangent function.

Finally, the magnitude of the thrust force needed (which can be provided by both propulsion systems) is simply $f=m a$. Note that if no solar sail is used, then the thrust shall be provided by the SEP system completely, and therefore the controls are simply $T=f, \alpha_{T}=\alpha_{a}, \delta_{T}=\delta_{a}$.

In general, the SEP engine can provide a maximum amount of thrust $T_{\max }$. If at any time $F>T_{\max }$, then the selected orbit cannot be followed with a pure SEP system: either another obit is to be chosen, or an additional propulsion system (e.g. sail) is to be used. It can also be noted that the mass of the spacecraft is not constant throughout the orbit, but is a function of time, and can be computed knowing the previous control history.

SEP-only solutions are not optimal for the hybrid spacecraft, in terms of propellant mass consumption, since they do not exploit the solar sail. A different approach can be used: since the kinematics is given, and so is the total acceleration, the attitude of the solar sail can be found, such that the SEP thrust is minimum at each instant of time.

The magnitude of the thrust can be expressed as a function of the sail cone and clock angles:

$$
a_{T}(\alpha, \delta)=\left|\mathbf{a}-a_{s} \hat{\mathbf{m}}\right|
$$

in which $\mathbf{a}$ is known through (24), and the term $a_{s} \hat{\mathbf{m}}$ can be computed using Eqs. (13), (15), and (17), and depends on the sail cone and clock angles, and the spacecraft mass. Therefore, at each point in the trajectory, and for a given mass, the sail angles $\alpha, \delta$ can be determined by solving the NLP problem:

$$
\left[\begin{array}{l}
\alpha^{*} \\
\delta^{*}
\end{array}\right]=\underset{\substack{0 \leq \alpha \leq \pi / 2 \\
0 \leq \delta<2 \pi}}{\arg \min } a_{T}(\alpha, \delta)
$$

If the mass as a function of time is known, then for each point on a given trajectory, the controls can be computed by solving problem (28). Figure 6a shows the surface $a_{T}(\alpha, \delta)$ for a specified position, velocity and mass of the spacecraft.

It was analytically shown in Ref. 17 that the minimum-thrust solution is such that, in the general case:

$$
\delta^{*}=\delta_{a}
$$

and since $\delta_{a}$ is known through (27), the problem can be reduced to:

$$
\alpha^{*}=\underset{0 \leq \alpha \leq \pi / 2}{\arg \min } a_{T}\left(\alpha, \delta_{a}\right)
$$



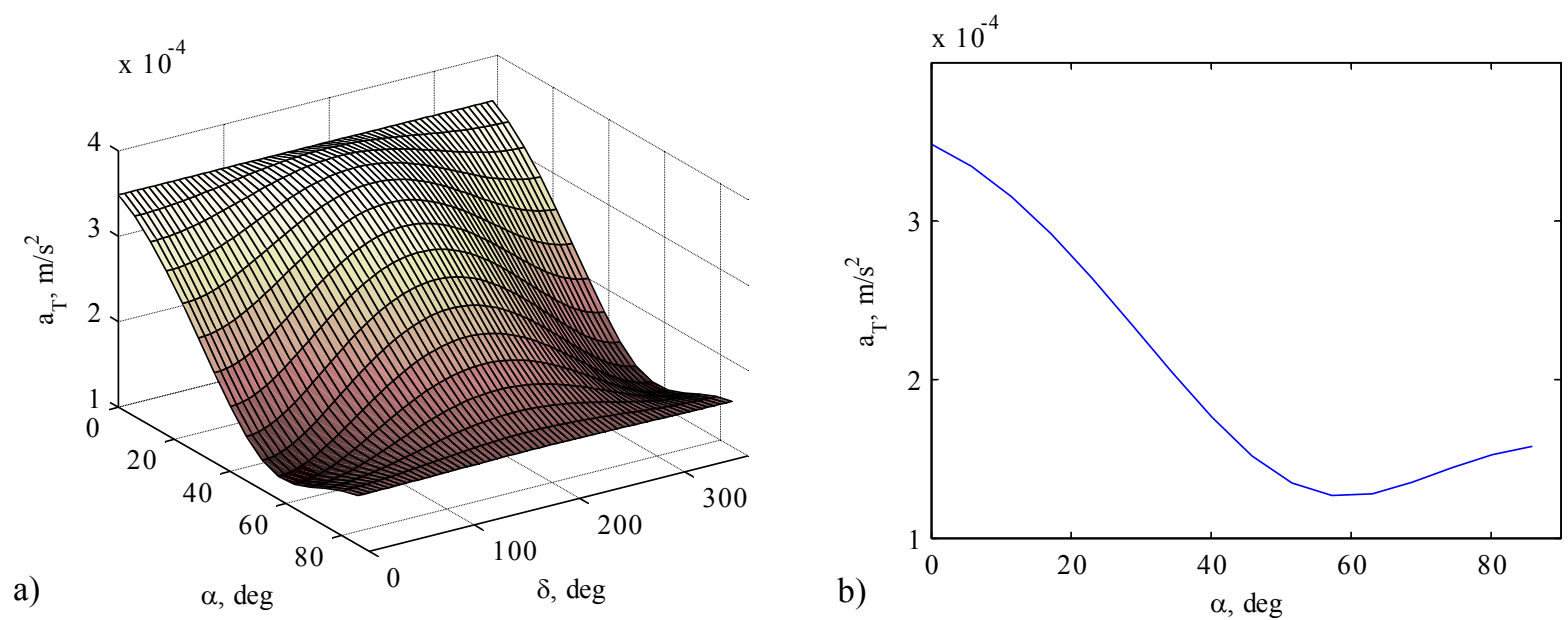

Fig. 6. At point $r=[1.006,0,0.0137] \mathrm{AU}$ and $\mathrm{v}=[0,-0.1781,0] \mathrm{km} / \mathrm{s}$ : (a) $\mathrm{SEP}$ acceleration as a function of the sail cone and clock angles, for a specific point on a given orbit. (b) Section of the previous surface for the optimal value of sail clock angle.

Figure $6 \mathrm{~b}$ shows the function $a_{T}\left(\alpha, \delta_{a}\right)$ for the same case as Fig. 6a. The minimization (30) is solved numerically, using an SQP method ${ }^{32}$ implemented in MATLAB ${ }^{\circledR}$ function fmincon. To prevent the possibility of converging to a local minimum, 4 different starting points, spread in the interval $[0, \pi / 2]$ are used.

Once $\alpha^{*}, \delta^{*}$ are found, the other controls can be easily computed: the modulus of the SEP thrust $T$ is simply given by $a_{T} m$; its direction can be found considering the SEP acceleration vector $\mathbf{a}_{T}=\mathbf{a}-a_{s} \hat{\mathbf{m}}$, that can be expressed in frame $(B)$ and then the angles can be found through Eq. (27) applied to $\mathbf{a}_{T}$.

The method that is used here to find the acceleration at each specific point $\mathbf{r}(t)$ can be extended to find entire orbits. This will be the subject of the following subsection.

\section{B. Extension to Periodic Orbits}

Here one-year periodic pole-sitter orbits are sought. The initial mass of the spacecraft $m_{0}$, at time $t_{0}=0$, the winter solstice, is assumed to be $1000 \mathrm{~kg}$. Note that this mass in general is not the launch mass, but the mass remaining after performing the transfer and injection into the pole-sitter orbit. This part of the mission is not subject of this paper. The controls at successive instants of time, together with the mass consumption, can be approximated by discretizing the trajectory into several points, each of which is spaced by a time interval $\Delta t$. At each point, controls can be estimated through (28). Once the thrust is known, it can be can assumed that this level of thrust remains almost constant for an interval $\Delta t$. Therefore the differential equation for the mass in system (22) can be integrated from $i^{\text {th }}$ time instant $t_{i}$ to the following one $t_{i}+\Delta t=t_{i+1}$ :

$$
m_{i+1}=m_{i}-\frac{T}{v_{e}} \Delta t
$$

Algorithm 1 is used to compute the entire one-year periodic orbit. The final time $t_{f}$ coincides with $2 \pi$ in the non-dimensional time frame.

\section{Constant Distance}

Firstly, the analysis with a pole-sitter orbit that keeps a constant distance from the Earth's pole is considered (Fig. 7). The kinematics of this trajectory is simply:

$$
\mathbf{r}(t)=\left[\begin{array}{c}
d \sin \delta_{e q} \cos \omega t+(1-\mu) \\
-d \sin \delta_{e q} \sin \omega t \\
d \cos \delta_{e q}
\end{array}\right]
$$

12

American Institute of Aeronautics and Astronautics 
Algorithm 1. Shape-based approach for computing controls.

\begin{tabular}{|ll|}
\hline 1: & Set $t=t_{0} ; m=m_{0}$ \\
3: & Do \\
4: & Given $\mathbf{r}(t), \dot{\mathbf{r}}(t), \ddot{\mathbf{r}}(t)$ from kinematics, \\
& find total acceleration using (25) and (27) \\
5: & Find $\alpha^{*}, \delta^{*}$ with (29) and solving problem (30) \\
5: & Find other controls $T, \alpha_{T}, \delta_{T}$ \\
6: & Update mass with $(31): m \leftarrow m-T \Delta t / v_{e}$ \\
7: & Update time: $t \leftarrow t+\Delta t$ \\
8: & While $t \leq t_{f}$ \\
\hline
\end{tabular}

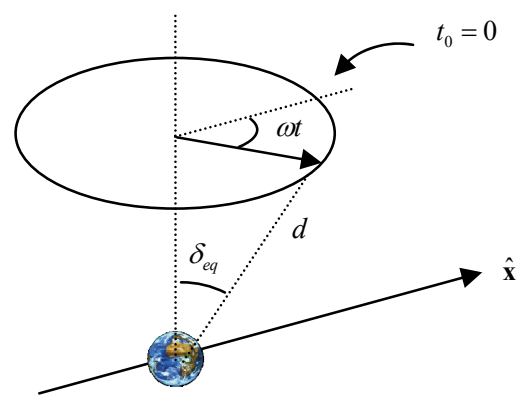

Fig. 7. The pole-sitter orbit with constant distance from the Earth.

and the derivatives are:

$$
\dot{\mathbf{r}}(t)=\left[\begin{array}{c}
-\omega d \sin \delta_{e q} \sin \omega t \\
-\omega d \sin \delta_{e q} \cos \omega t \\
0
\end{array}\right] ; \ddot{\mathbf{r}}(t)=\left[\begin{array}{c}
-\omega^{2} d \sin \delta_{e q} \cos \omega t \\
\omega^{2} d \sin \delta_{e q} \sin \omega t \\
0
\end{array}\right]
$$

Using Algorithm 1, the optimal hybrid solution for the constant-distance pole-sitter is obtained. Figure 8 shows the trajectory of the spacecraft over one year, superimposed on the cone described by the Earth's polar axis. The bold arrows are proportional to the total local acceleration from Eq. (24), that needs to be counterbalanced by the total thrust, for maintaining the orbit. The other vector fields refer to the reference case $\beta_{0}=0.05$. The gray arrows represent the attitude of the solar sail, $\hat{\mathbf{n}}$. Their direction does not exactly match the direction of the force produced by the solar sail (black arrows in figure), due to the non-ideal sail that was considered. Finally, the black, non-bold arrows represent the SEP thrust. It can be noted that the gravitational acceleration is mostly directed towards $-\hat{\mathbf{z}}$, therefore the resulting thrust shall be in the opposite direction. The solar sail thrust has a component in $+\hat{\mathbf{z}}$, but it is accompanied by a significant component along $+\hat{\mathbf{x}}$, i.e. facing away from the Sun. The SEP is therefore providing the missing component in $+\hat{\mathbf{z}}$, but also counteracting the residual $\hat{\mathbf{y}}$ component.

Figure 9 shows the magnitude of the acceleration provided by the sail (continuous lines) and SEP (dotted lines), for different values of $\beta_{0}$ : the solid lines refer to $\beta_{0}=0$, i.e. a pure SEP system with no sail, the long-dashed lines to $\beta_{0}=0.05$ and the short-dashed lines to $\beta_{0}=0.1$. The bold circled line is the magnitude of the gravitational forces. In the case of pure SEP, the SEP acceleration is obviously the same as the gravitational acceleration. In the hybrid case, clearly the SEP acceleration is lower for higher values of $\beta_{0}$. Additionally, from the figure it is possible to see that higher acceleration is required from SEP around the summer solstice, roughly in the interval $t \in[100,250] \mathrm{d}$.

Figure 10 and Fig. 11 show respectively the cone and clock angles of both the sail and the SEP acceleration. In the case $\beta_{0}=0$, the sail cone has no meaning. The trend of the clock angles of both the sail and the SEP is the same, and does not depend on the spacecraft mass or initial lightness number. Their shape is simply sinusoidal during one year: therefore, these two controls will not be shown in the rest of this work. 


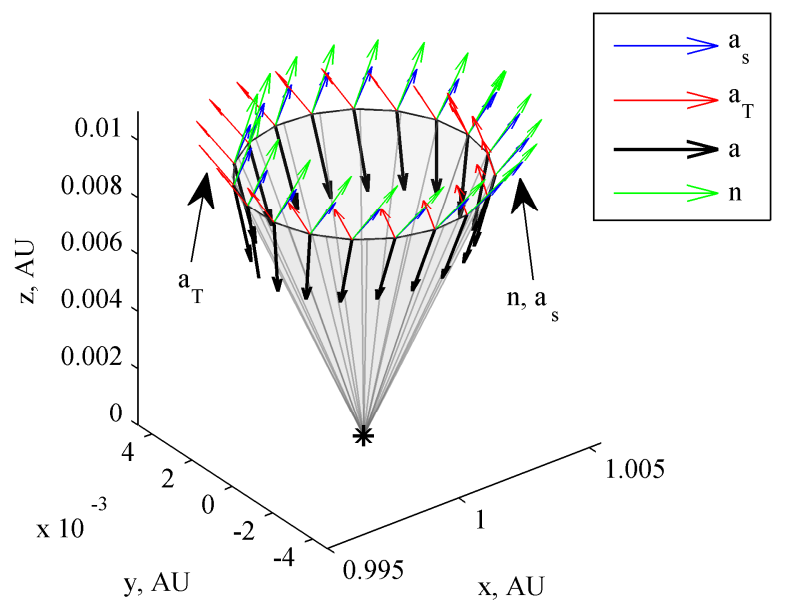

Fig. 8. Trajectory and thrust vectors for $\beta_{0}=0.05$ over one year on a flat orbit $d=0.01 \mathrm{AU}$.

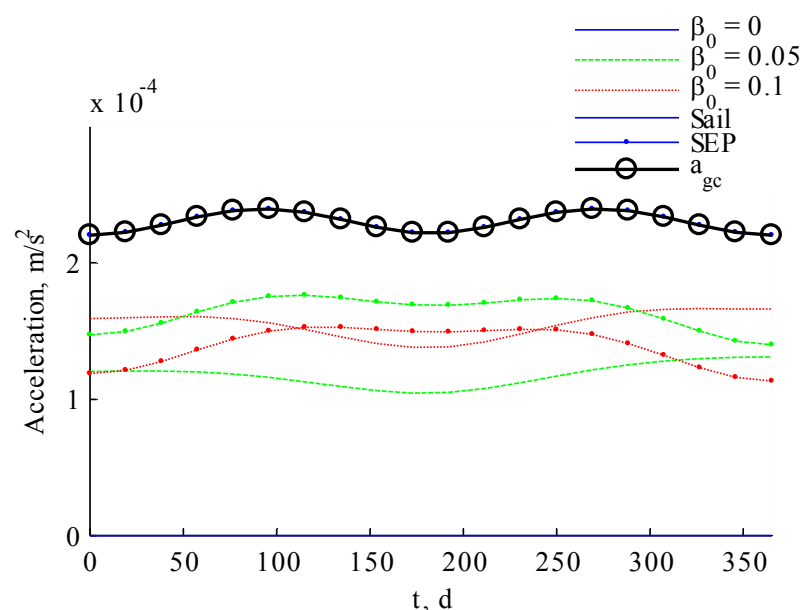

Fig. 9. Acceleration of the sail (non-dotted lines) and SEP (dotted lines) for different values of $\beta_{0}$, over one year on a flat orbit $d=0.01 \mathrm{AU}$.

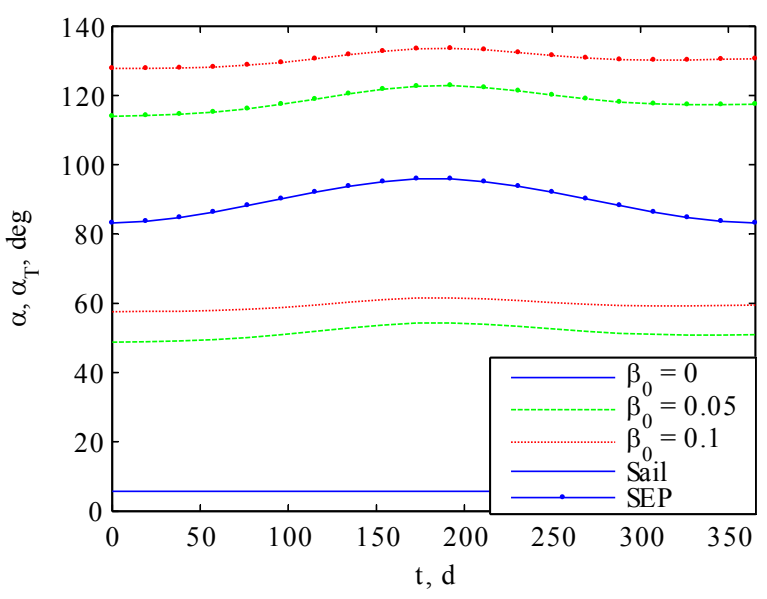

Fig. 10. Cone angles of sail (non-dotted lines) and SEP thrust (dotted lines) for different values of $\beta_{0}$, over one year on a flat orbit $d=0.01 \mathrm{AU}$. The angles have the same value point-wise; hence all the lines are overlapped.

Figure 12 shows the spacecraft mass change. In particular the figure shows the mass gain of the hybrid propulsion with respect to the pure SEP spacecraft.

A parametric analysis can be done, for all the orbits maintaining constant distance from the Earth, as a function of the distance itself. Figure 13 shows the propellant mass fraction of the spacecraft in a constant-distance pole-sitter orbit, for one year, as a function of the distance of the orbit, for three values of $\beta_{0}$. These orbits become extremely expensive when the distance from the Earth decreases, and the Earth's gravitational attraction becomes predominant with respect to the other forces of the CR3BP. This result matches what was found in Ref. 24. It is also interesting to note that the propellant consumption has a minimum for a value of $d \cong 0.017 \mathrm{AU}$.

Finally, Fig. 14 shows the propellant mass fraction needed for a spacecraft of $1000 \mathrm{~kg}$ to maintain an orbit at $d=0.015 \mathrm{AU}$ from the Earth. This graph is relevant because it highlights that a consistent gain in propellant mass is obtained by adding a small sail to the pure SEP spacecraft: in fact, the slope of the curve is highest for small values of $\beta_{0}$. As the lightness number increases towards very high values, the gain in propellant mass for a given increase of $\beta_{0}$ becomes less. However, this graph justifies the research on the hybrid spacecraft, seen as a pure SEP system with a small auxiliary sail. The trend of this graph is the same for different values of $d$. The effect of the sail on the total mass budget of the sail will be discussed later.

14

American Institute of Aeronautics and Astronautics 


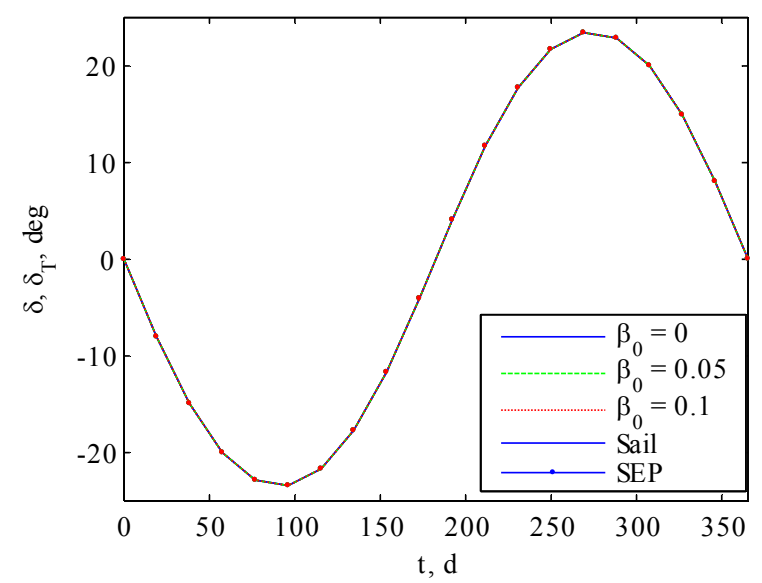

Fig. 11. Sail and SEP thrust clock angles for different values of $\beta_{0}$, over one year on a flat orbit $d=0.01 \mathrm{AU}$. The angles have the same value pointwise; hence all the lines are overlapped.

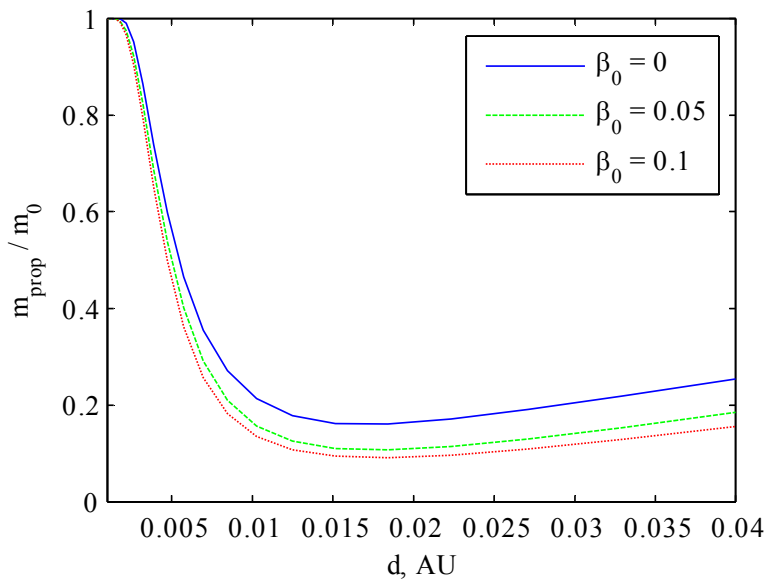

Fig. 13. Propellant mass fraction needed for one year on a flat, circular orbit, as a function of the distance $d$ from Earth (initial spacecraft mass $m_{0}=1000 \mathrm{~kg}$ ).

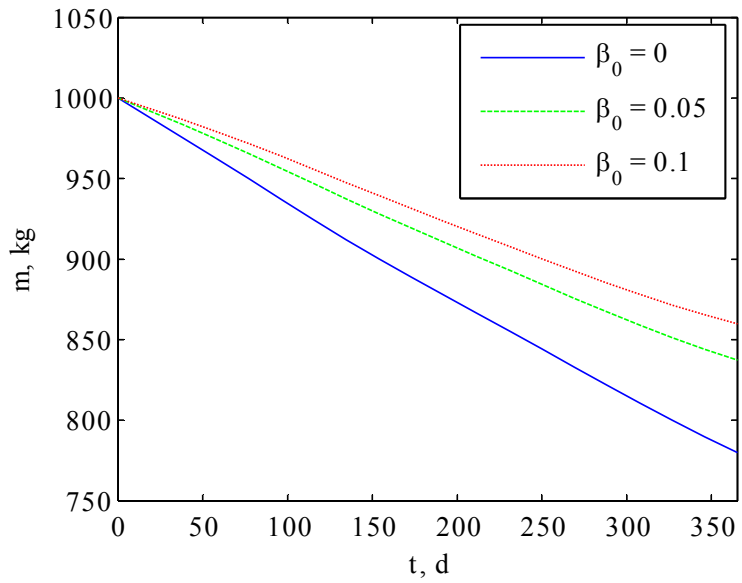

Fig. 12. Mass trend for different values of $\beta_{0}$, over one year on a flat orbit $d=0.01 \mathrm{AU}$.

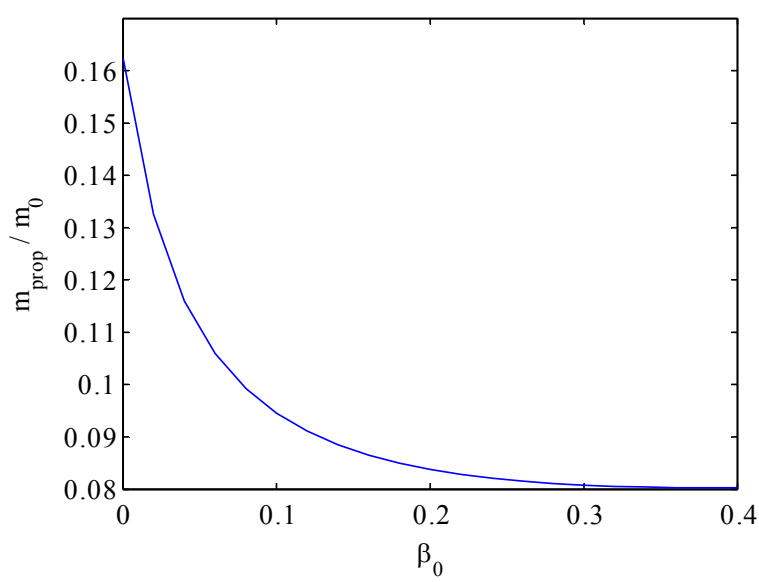

Fig. 14. Propellant mass fraction needed for one year on a flat, circular orbit $(d=0.015 \mathrm{AU})$, as a function of the sail lightness number $\beta_{0}$ (initial spacecraft $\left.\operatorname{mass} m_{0}=1000 \mathrm{~kg}\right)$.

Note that even if a higher $\beta_{0}$ sail produces a higher acceleration $\mathbf{a}_{s}$, its direction is still constrained and related to the cone angle, and in general it is not aligned to the total acceleration vector a (see vectors in Fig. 8). Therefore, a higher sail acceleration is in general accompanied by a greater acceleration component in an unwanted direction, which shall be compensated by using the thruster.

\section{Tilted Orbits}

Different families of pole-sitter orbits, which reduce propellant mass, can be obtained by relaxing the constraint of a constant distance from the Earth. It is possible to reduce the thrust and better exploit the capabilities of the solar sail by tilting the orbit such that the required acceleration is towards the Sun, and thus it can be counterbalanced more efficiently by the solar sail.

A different set of shapes, in which we introduce as parameters the distance from the Earth at winter solstice $d_{1}$ and at summer solstice $d_{2}$ (Fig. 15), will now be considered. The $\hat{\mathbf{z}}$ coordinate is varied between these two values with a sinusoidal law: 


$$
\begin{gathered}
d(t)=d_{1}+\left(d_{2}-d_{1}\right) \frac{\cos (\omega t+\pi)+1}{2} \\
\mathbf{r}(t)=\left[\begin{array}{c}
d(t) \sin \delta_{e} \cos \omega t+(1-\mu) \\
-d(t) \sin \delta_{e} \sin \omega t \\
d(t) \cos \delta_{e}
\end{array}\right]
\end{gathered}
$$

If for example $d_{1}=0.01 \mathrm{AU}$ and $d_{2}=0.015 \mathrm{AU}$ are selected, the orbit in Fig. 16 is obtained. From the vector fields of the forces, it can be seen that during summer the alignment of the gravitational forces is more favorable.

The plot of the accelerations as function of time (Fig. 17) confirms this: while the sail provides more or less a constant acceleration through the year, the SEP acceleration drops considerably around summer. This results in significant saving in propellant mass.

Figure 18 shows the cone angles, while Fig. 19 represents the mass as a function of time. This is to be compared to Fig. 12: the mass saving due to the tilting of the orbit is evident, although the average distance of the spacecraft from the Earth during an orbit is higher. It is worth noting that the propellant mass saving is not only due to the increased distance of the spacecraft during summer, but also to the favorable alignment of the forces. In fact, tilting the orbit in the other direction (i.e. $d_{1}=0.015 \mathrm{AU}$ and $d_{2}=0.01 \mathrm{AU}$ ) produces a much worse result in terms of propellant consumption. Therefore it can be concluded that, in general, it is cheaper to observe the North Pole from a shorter distance in winter than in summer.

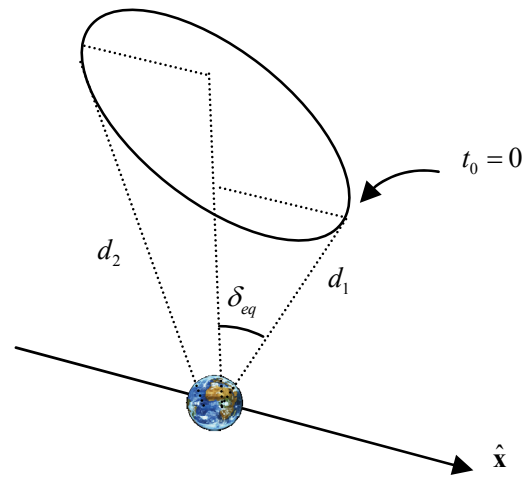

Fig. 15. Definition of $d_{1}$ and $d_{2}$, and origin for the tilted, shape-based orbits.

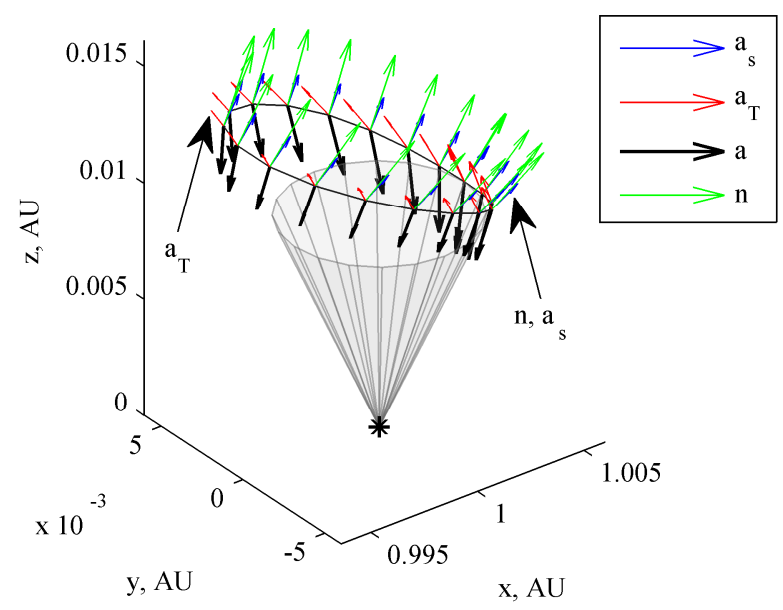

Fig. 16. Trajectory and thrust vectors for $\beta_{0}=0.05$ over one year on a tilted orbit $d_{1}=0.01 \mathrm{AU}, d_{2}=$ 0.015 AU.

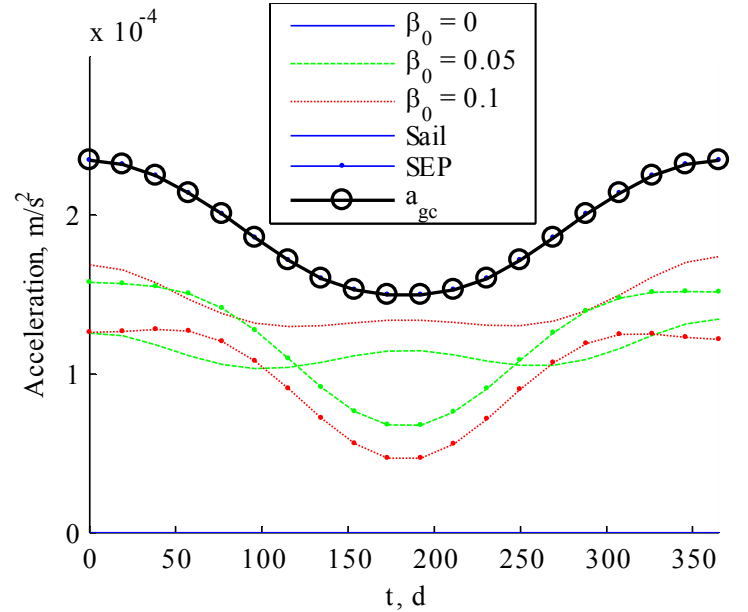

Fig. 17. Acceleration of the sail (non-dotted lines) and SEP (dotted lines) for different values of $\beta_{0}$, over one year on a tilted orbit $d_{1}=0.01 \mathrm{AU}, d_{2}=0.015 \mathrm{AU}$. 


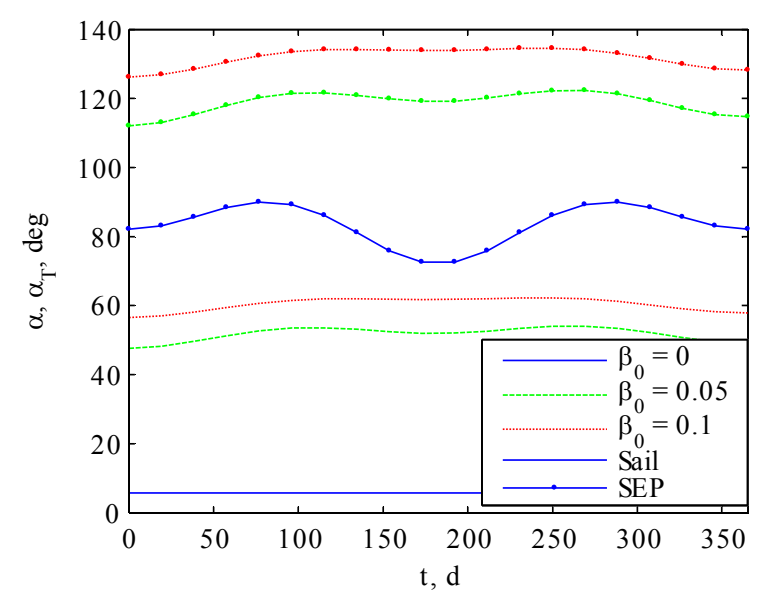

Fig. 18. Sail and SEP cone angles for different values of $\beta_{0}$, over one year on a tilted orbit $d_{1}=0.01 \mathrm{AU}, d_{2}=$ 0.015 AU.

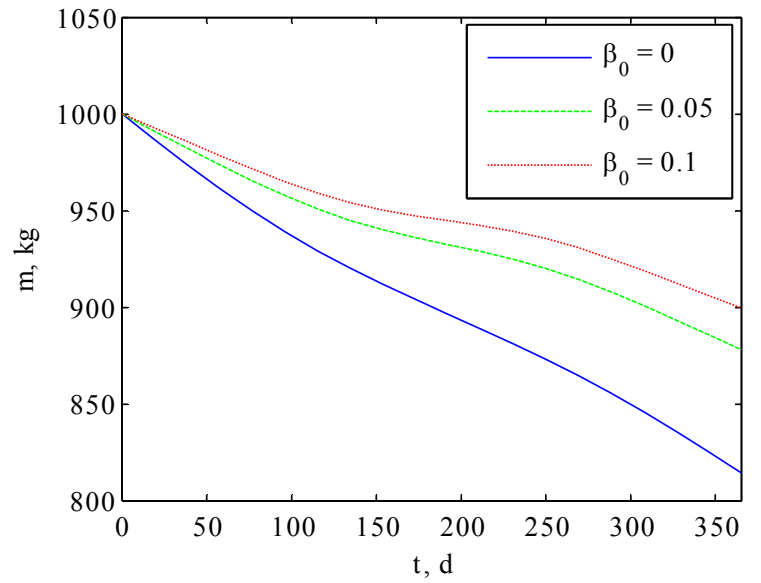

Fig. 19. Mass trend for different values of $\beta_{0}$, over one year on a tilted orbit $d_{1}=0.01 \mathrm{AU}, d_{2}=0.015 \mathrm{AU}$.

\section{Remarks}

The solutions generated with the proposed shape-based method are optimal, in the sense that the thrust to maintain a given orbit is minimized at each instant of time. Therefore, for the specific paths considered, the control profile that was found is the one that allows minimum propellant consumption. If, for example, a payload requires a constant distance from the Earth throughout the year, then the orbit is defined. Note that in the case where the kinematics is fully determined, it was verified by several numerical experiments that Algorithm 1 provides solutions that are extremely similar to those that can be found by solving an optimal control problem, minimizing the propellant mass, and keeping the orbit fixed. However, if there is no constraint or requirement on the orbit, but for example only a range of distance is specified (depending for example on the instrument requirements) then the use $a$ priori of a specific orbit cannot be justified.

It was shown that different orbits could affect the amount of thrust needed at each instant of time, and thus the propellant mass over one year.

Due to the constraint of the pole-sitter spacecraft, all possible orbits can be described through the distance from the Earth as a function of time during one year, $d(t)$. Among these, there shall be at least one particular 1-yearperiodic orbit, for any given value of $\beta_{0}$, that minimizes propellant mass while maintaining the spacecraft above the pole.

As seen in Fig. 13, the propellant mass is not monotonically increasing with the distance from the Earth, but it has a maximum. Although the figure refers to the case of flat orbits, the same trend is seen for tilted orbits. This fact indicates that orbits do not become indefinitely cheap while increasing the distance. Therefore, it is expected to find optimal orbits without limiting the maximum distance from Earth.

If the kinematics of the orbit is not known a priori (that is equivalent to say that the distance function $d(t)$ is not given), then the semi-analytical procedure used so far is not applicable, and the solution, that includes determining the optimal trajectory, is found by solving an optimal control problem. The optimal control problem is one of finding the control history of a given dynamical system, such as to optimize a given cost function, while satisfying the dynamics itself and possibly other constraints, which can include initial/final conditions.

In the following section, the solution will be found through a direct method. All direct methods need to be initialized with an initial (or first-guess) solution, which is close enough to the optimal one to guarantee convergence; orbits and control histories found with the shape-based method will be used for this purpose.

\section{IV.Pseudo-Spectral Methods}

In general, an optimal control problem can be defined in the following way. Given a dynamical system, described by a set of differential equations for the states $\mathbf{x}$, from initial time $t_{0}$ to final time $t_{f}$, 


$$
\dot{\mathbf{x}}(t)=\mathbf{f}(\mathbf{x}(t), \mathbf{u}(t), t) \in \mathbb{R}^{n_{x}}, \quad t \in\left[t_{0}, t_{f}\right]
$$

the problem is to find the control law $\mathbf{u}(t) \in \mathbb{R}^{n_{u}}$, such as to minimize the cost function:

$$
J=\varphi\left(\mathbf{x}_{0}, \mathbf{x}_{f}, t_{0}, t_{f}\right)+\int_{t_{0}}^{t_{f}} L\left(\mathbf{x}\left(t^{\prime}\right), \mathbf{u}\left(t^{\prime}\right), t^{\prime}\right) \mathrm{d} t^{\prime} .
$$

The cost function is split into two addends. The first one, the endpoint cost, is a function of the initial and final states and time only. The second, under the integral and a function of time, $L(\mathbf{x}(t), \mathbf{u}(t), t)$, is the Lagrangian of the system. The initial and final time can also be free, as optimization parameters. Additional non-linear constraints can also be introduced, of the type:

$$
\mathbf{c}(\mathbf{x}, \mathbf{u}, t) \leq 0
$$

These constraints include path constraints, bounds on the states, the controls, and feasible intervals for the initial and final states.

Direct methods for optimal control problems find the solution of an optimal control problem by discretizing the time interval into a finite number of points (collocation points or nodes) and then transcribing the finite-dimensional problem into a constrained non-linear programming (NLP) problem that can be solved with one of the several methods available in literature. Therefore the controls and states over time are completely described by a finite set of points, and the continuous time history is reconstructed from the collocation points through interpolating functions. In this work, pseudo-spectral methods will be used: Pseudo-spectral methods have been used to tackle partial differential equations ${ }^{33}$ and have been applied in many areas of science, engineering and modeling. More recently, starting from about 1990, they have been also used to solve optimal control problems. ${ }^{34-35}$

They are based on Legendre or Chebyshev polynomials for interpolation and approximation of the derivatives and integrals.

The advantage of using pseudo-spectral methods for approximating a continuous and smooth function is that the derivative of the function at the collocation points and the integral of the function can be computed through matrix multiplication only, ${ }^{36}$ and do not need to be approximated with finite differences. This comes into use for computing the derivatives of the states in Eq. (36) and the integral of the Lagrangian of the cost function in Eq. (37).

A particular implementation of pseudo-spectral methods was selected for this work. The tool, named PSOPT, was created and coded in $\mathrm{C}++$ by Becerra $^{36}$ and is free and open source. PSOPT can deal with endpoint constraints, path constraints, and interior point constraints. Bounds on the states and controls can be enforced, as well as intervals for initial and final states. Furthermore, it makes use of the ADOL-C library for the automatic differentiation of $\mathrm{C}++$ code. It allows the machine to compute automatically the gradients and sparse Jacobians of the user-supplied dynamics and constraint functions required by PSOPT. The NLP problem is solved through IPOPT,${ }^{37}$ an open source $\mathrm{C}++$ implementation of an interior point method for large scale problems.

\section{A. Pole-Sitter Optimal Control Problem}

The problem is now to find optimal periodic orbits with respect to a given cost function, specifically to minimize the propellant consumption, or maximize the final mass.

Given that a pole-sitter orbit is fully determined through the function $d(t)$, a possible approach is to obtain an equation for the dynamics of $d(t)$. If the position vector is expressed through Eq. (23), then the three-component dynamics for $\mathbf{r}(t)$ (20) can be transformed into three scalar equations, all involving $d(t), \dot{d}(t), \ddot{d}(t)$. One of these equations can be used for describing the dynamics of $d$, while the other two represent differential constraints on the control, necessary for meeting the pole-sitter condition (i.e. maintain the spacecraft above the pole). In this work, rather than using this approach, the dynamics of $\mathbf{r}(t)$ (22) are used, and then the pole-sitter constraints are enforced explicitly, to ensure that $\mathbf{r}(t)$ is compatible with Eq. (23). Therefore, the dynamical system is the one in Eq. (22), and the state vector for the problem is:

$$
\mathbf{x}=\left[\begin{array}{lllllll}
r_{x} & r_{y} & r_{z} & v_{x} & v_{y} & v_{z} & m
\end{array}\right]^{T}
$$

with the following bounds, in non-dimensional units: 


$$
\mathbf{x}_{l}=\left[\begin{array}{c}
1-d_{\max } \sin \left(\delta_{e q}\right) \\
-d_{\max } \sin \left(\delta_{e q}\right) \\
0 \\
-0.1 \\
-0.1 \\
-0.1 \\
0
\end{array}\right], \quad \mathbf{x}_{u}=\left[\begin{array}{c}
1+d_{\max } \sin \left(\delta_{e q}\right) \\
d_{\max } \sin \left(\delta_{e q}\right) \\
d_{\max } \cos \left(\delta_{e q}\right) \\
0.1 \\
0.1 \\
0.1 \\
m_{0}
\end{array}\right] .
$$

It should be noted that some bounds are not strictly necessary for finding the optimal solution: for example, both the velocity and the mass states are automatically limited due to the dynamics of the problem. Nevertheless, bounds for the states and controls are necessary, and used by PSOPT to scale the problem. The position, instead, can be limited to a maximum distance from the Earth $d_{\max }$, for example as a requirement of the payload.

As the problem will be solved for one orbital period, initial and final times are fixed as:

$$
\begin{aligned}
& t_{0}=0 \\
& t_{f}=2 \pi
\end{aligned}
$$

The following fixed initial states are considered:

$$
\begin{aligned}
& r_{y}=0 \\
& v_{x}=0 \\
& v_{z}=0 \\
& m(0)=m_{0}
\end{aligned}
$$

The first condition, together with proper bounds on $r_{x}, r_{z}$, guarantees that the starting point is at the winter solstice. The conditions on the velocity represent the fact that the orbit shall be symmetric with respect to the $\hat{\mathbf{x}}-\hat{\mathbf{z}}$ plane. Other initial states are free within the state bounds. Therefore, the optimizer is allowed to move the initial point on the orbit, specifically raise or lower it, as well as the initial velocity.

The periodicity of the solution is set requiring that the final states, except the mass, are the same as the corresponding initial states:

$$
\begin{aligned}
& \mathbf{r}\left(t_{f}\right)=\mathbf{r}(0) \\
& \mathbf{v}\left(t_{f}\right)=\mathbf{v}(0)
\end{aligned}
$$

Note that, despite the choice of the winter solstice as the initial point, since the orbit is periodic, it does not imply that the injection from transfer should necessarily happen in this point.

Since the spacecraft has to stay along the polar axis of the Earth at each time $t \geq t_{0}$, two path constraints are introduced, such that:

$$
\begin{aligned}
& \operatorname{atan} 2\left(-r_{2, y}, r_{2, x}\right)-\omega t=0 \\
& \sqrt{r_{2, x}^{2}+r_{2, y}^{2}}-r_{2, z} \tan \left(\delta_{e q}\right)=0
\end{aligned}
$$

The former defines the angular position of the spacecraft around the Earth in the $\hat{\mathbf{x}}-\hat{\mathbf{y}}$ plane, while the latter constrains the distance of the spacecraft measured from an axis passing from the centre of the Earth and parallel to $\hat{\mathbf{z}}$ (see Fig. 20).

Controls are transformed into Cartesian coordinates, to prevent problems with ambiguity and periodicity of angular variables, which can arise (see Ref. 38, pp. 140). Therefore, the thrust is described through its Cartesian components along $\hat{\mathbf{r}}_{1}, \hat{\boldsymbol{\theta}}_{1}, \hat{\boldsymbol{\varphi}}_{1}$, respectively $T_{r_{1}}, T_{\theta_{1}}, T_{\varphi_{1}}$ The attitude of the solar sail is analogously described through the components in the same frame of the unit vector $\hat{\mathbf{n}}$. Therefore the vector of controls is:

$$
\mathbf{u}=\left[\begin{array}{llllll}
n_{r_{1}} & n_{\theta_{1}} & n_{\varphi_{1}} & T_{r_{1}} & T_{\theta_{1}} & T_{\varphi_{1}}
\end{array}\right]^{T}
$$




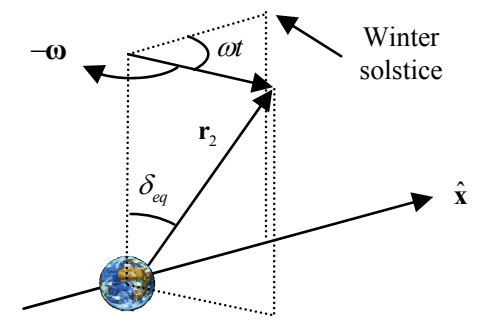

Fig. 20. Origin of time and angular velocity of the pole-sitter.

Bounds for the controls are defined as:

$$
\begin{aligned}
& \mathbf{u}_{l}=\left[\begin{array}{llllll}
-1 & -1 & -1 & -T_{u} & -T_{u} & -T_{u}
\end{array}\right]^{T} \\
& \mathbf{u}_{u}=\left[\begin{array}{llllll}
+1 & +1 & +1 & T_{u} & T_{u} & T_{u}
\end{array}\right]^{T}
\end{aligned}
$$

The use of Cartesian components forces the use of additional path constraints, for limiting the magnitude of each vector. The norm of the SEP thrust is in fact limited by the capabilities of the engine:

$$
\sqrt{T_{r_{1}}^{2}+T_{\theta_{1}}^{2}+T_{\theta_{1}}^{2}} \leq T_{u}
$$

Concerning the attitude of the sail, the three components $n_{r_{1}}, n_{\theta_{1}}, n_{\varphi_{1}}$ are sufficient to determine the direction of $\mathbf{n}$, and its magnitude is not relevant. Even though a path constraint is enforced, to guarantee the uniqueness of the solution:

$$
\sqrt{n_{r_{1}}^{2}+n_{\theta_{1}}^{2}+n_{\theta_{1}}^{2}}=1 \text {. }
$$

Different cost functions will be used and will be specified in the relevant subsection.

\section{B. Results}

The optimized solutions presented here are for the three values of $\beta_{0}=0,0.05,0.1$.

The following results use 60 collocation points in the 1 year mission duration. It was assessed that a higher number of points did not result in any significant change in the states or controls history. The convergence of PSOPT is easier and faster using a small number of points: therefore, all the following solutions were found by iteratively increasing the number of points, from 20 to 60 , and using the optimal solution found at one iteration as a first guess for the following one.

The following sub-section will present optimal orbits that minimize the propellant consumption of the spacecraft over one period, with no constraints on the spacecraft distance from the Earth. In the following, instead, a trade-off will be presented, showing the additional propellant needed to satisfy given constraints on the distance.

\section{Minimum Propellant Consumption}

In this section, propellant consumption will be minimized; therefore the cost function is simply:

$$
J=-m_{f}=-m\left(t_{f}\right)
$$

that is, to maximize the final mass after one period, or one year. Note that linear minimum-fuel problems, with constrained control magnitude, usually result in a bang-off-bang control (see Ref. ${ }^{39}$, pp. 298-307). In this case, such a control is not expected due to the path constraints on the dynamics: in general, a continuous control is needed to maintain a pole-sitter orbit. Even if a limitation on the maximum magnitude of the thrust is taken into account, the SEP thrust law does not show an on-off structure. However, we will show that the SEP system could be switched off in some arcs, if the solar sail is sufficient to maintain the pole-sitter condition.

In this section constraints are not enforced on the distance of the spacecraft from the Earth. The value $d_{\max }$ was set to the rather high value of $0.1 \mathrm{AU}$. The aim is to find the cheapest orbits in terms of propellant consumption.

Figure 21 shows the three different optimal trajectories, obtained with PSOPT, for each value of $\beta_{0}$, and Fig. 22 shows the distance of the spacecraft form the Earth. The first point to note is that the pure SEP spacecraft would optimally follow an orbit that is symmetric in summer and winter. On the other hand, the optimal orbits gets closer to the Earth in winter and farther in summer, as the lightness number of the solar sail increases. The distance can even double from winter to summer for a solar sail with a lightness number of 0.1 . 

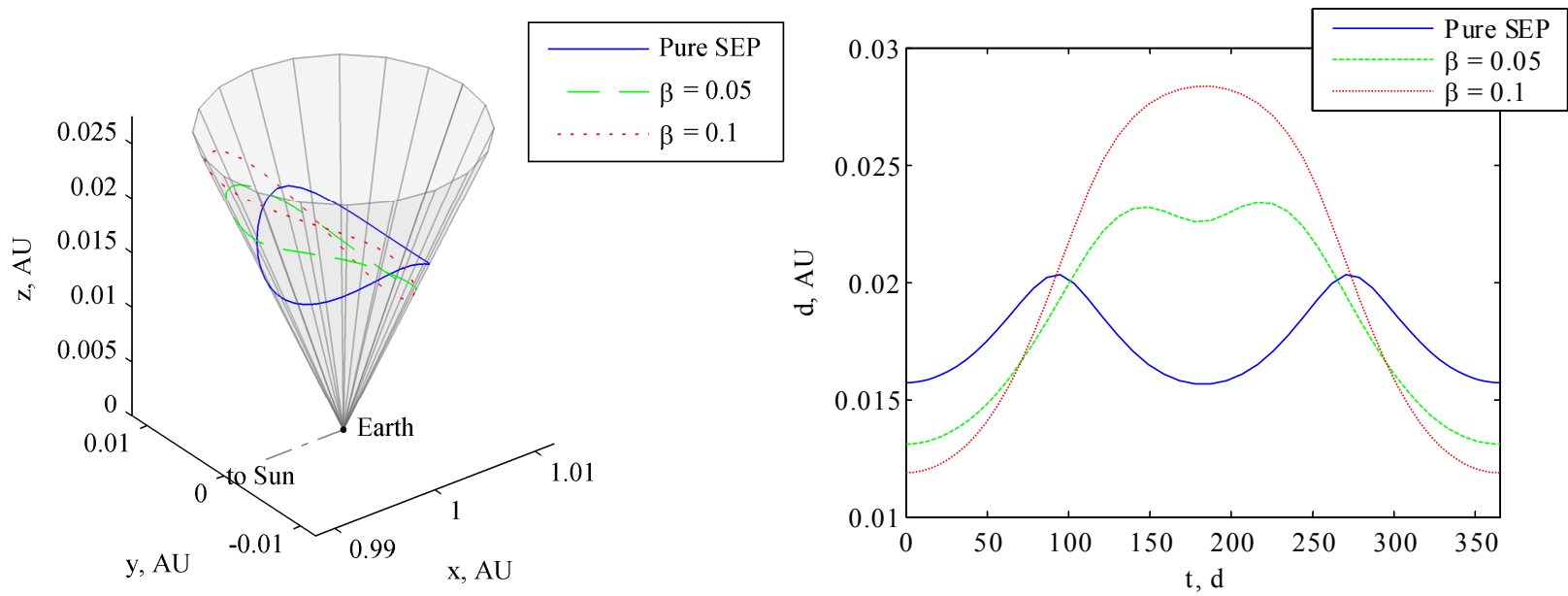

Fig. 21. Optimal hybrid pole-sitter orbits, minimizing the propellant mass over one period, for three different values of $\beta_{0}$, during one year.

Fig. 22. Distance from Earth centre $(d)$ for three different values of $\beta_{0}$, during one year.

Figure 23 shows the acceleration provided by the solar sail and the SEP system. Considering the pure SEP case in the analysis, two thrust regions can be identified: one across the summer solstice and one across the winter solstice. Due to the selected time-frame, starting at the winter solstice, the latter region is split in the plot. In these two regions the SEP acceleration is almost constant. The two regions are separated by two short arcs, in the autumn and spring equinoxes, in which the thrust becomes very low, and the spacecraft motion along $\hat{\mathbf{z}}$ is inverted: the instant with lowest thrust happens at the maximum distance from Earth (compare with Fig. 22).

If a solar sail is added (see the case $\beta_{0}=0.05$ in Fig. 23), the SEP acceleration required in the two thrust arcs decreases, and thrust region around the summer solstice becomes shorter, while two non-thrusted arcs expand, increasing the time when the thruster is off, and the solar sail is almost sufficient to maintain the spacecraft orbit.

Eventually, for a suitably large solar sail $\left(\beta_{0}=0.1\right)$, the thrust region around the summer solstice disappears, while the two ballistic arcs merge: becoming only one, lasting from spring to autumn approximately. Hence the tilting of the orbit can be explained in the following way: having the spacecraft high in summer and a low in winter allows the orbit to exploit, for a considerable amount of time, the solar sail and the natural forces only to drive the spacecraft back to the winter solstice point and close the orbit.

The mass as a function of time is represented in Fig. 24. In this figure the substantial saving in propellant that is given by a relatively small sail is evident: for a 1-year orbit, the propellant mass decreases from $158 \mathrm{~kg}$ (for the pure $\mathrm{SEP})$ to $97 \mathrm{~kg}\left(\beta_{0}=0.05\right)$. Confirming the result found before (see Fig. 13), a reduction of propellant mass is obtained by adding a relatively small sail. However, the saving in propellant mass is less than proportional to the lightness number.

Figure 25 shows the angle between the solar sail normal and the SEP thrust. The purpose of this plot is twofold. The first is to show that, throughout the whole mission, the required thrust is never lying in the plane of the solar sail. Thrusting along the sail would pose severe problems due to exhaust gases impinging on the sail itself. The second aim is to show that the rotation of the thrust vector with respect to the sail is limited. If it is assumed that the solar sail is fixed with the spacecraft, then its orientation can be changed by changing the attitude of the spacecraft. Figure 25 shows that the SEP thruster cannot be fixed with the spacecraft, as the thrust direction needs to vary; instead, it shall be mounted on a gimbal. The rotation needed is less than 2 degrees, in the worst case $\left(\beta_{0}=0.1\right)$, hence a simple mechanism can be sufficient to guarantee the required thruster pointing.

Finally, Table 1 summarizes some features of the three optimal orbits (for each considered value of $\beta_{0}$ ) that were designed. 

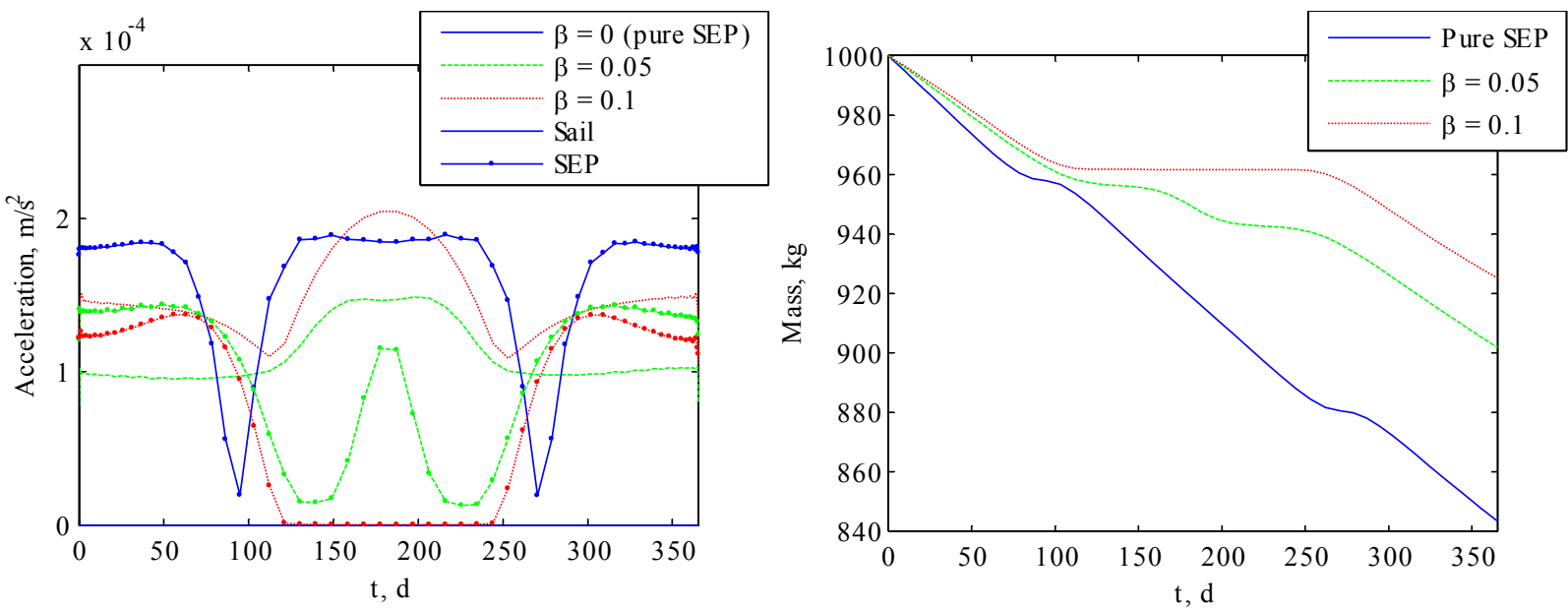

Fig. 23. Acceleration of the sail (non-dotted lines) and SEP (dotted lines) for three different values of $\beta_{0}$, during one year.

Fig. 24. Mass as a function of time, for three different values of $\beta_{0}$, during one year.

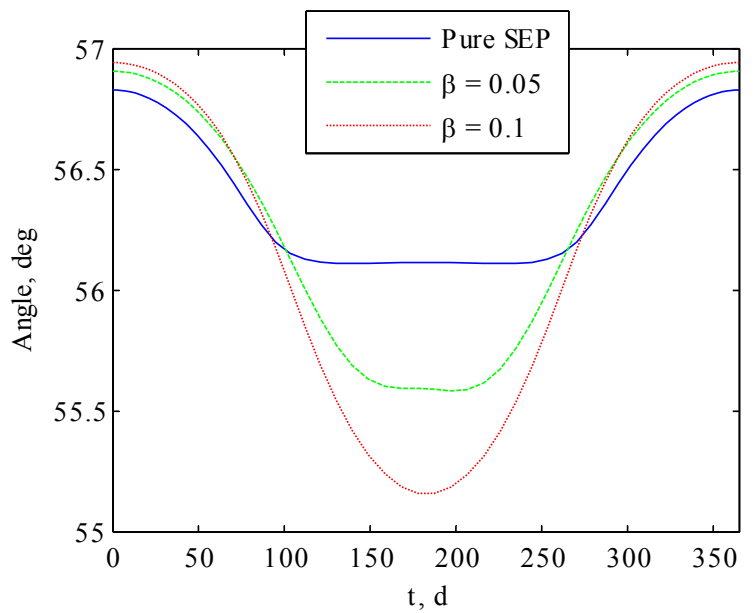

Fig. 25. Angle between the thrust vector $u_{T}$ with respect to the solar sail normal $n$, for three different values of $\beta_{0}$, during one year.

Table 1. Summary of characteristics of unconstrained optimal orbits for three different values of sail lightness number. Time frame is one year; initial mass is $m_{0}=1000 \mathrm{~kg}$.

\begin{tabular}{cccccc}
\hline \hline$\beta_{0}$ & $\min _{t} d(t), \mathrm{AU}$ & $\max _{t} d(t), \mathrm{AU}$ & $m_{f}, \mathrm{~kg}$ & $m_{\text {prop }} / m_{0}$ & $T_{\max }, \mathrm{N}$ \\
\hline 0.0 & 0.015675 & 0.020332 & 843.430417 & 0.156570 & 0.180648 \\
0.05 & 0.013116 & 0.023422 & 901.896219 & 0.098104 & 0.141085 \\
0.1 & 0.011896 & 0.028363 & 925.192867 & 0.074807 & 0.134256 \\
\hline \hline
\end{tabular}

2. Orbit Performance Trade-off

In this subsection the trade-off between an orbit with a particular feature, and the additional propellant mass needed to maintain it is considered.

A possible, important requirement of the pole-sitter spacecraft could be the maximum distance from the Earth. It has been shown that fuel-optimal orbits vary their distance quite consistently, and this can be an issue, for example, for guaranteeing a sufficient resolution for observation of the Earth. Therefore families of optimal orbits have been designed, constraining the maximum distance of the spacecraft, by gradually decreasing the value $d_{\max }$. 
Table 2 presents the results of the optimal orbits for different values of $d_{\max }$ and $\beta_{0}$. Note that for values of $d_{\max }$ lower than those considered in the table, the orbit is substantially flat, and therefore the optimal constrained solution coincides with the flat orbit found with the shape-based approach, and the value of the final mass after one year is the one represented in Fig. 13. Note that the thrust peak value does not change significantly as the orbit gets closer to the Earth, but the propellant needed is nevertheless more due to a higher value of the thrust required throughout the whole orbit. This is due to the fact that the peak of thrust happens at the beginning of the orbit, at the winter solstice, and when the spacecraft mass is highest. The distance of the orbits from Earth at the winter solstice is almost the same as long as $d_{\max }>0.013 \mathrm{AU}$, and so is the thrust needed at that point. An example of the families of orbits that are obtained are plotted in Fig. 26, for $\beta_{0}=0.05$.

Table 2. Summary of optimal orbits (minimum propellant mass consumption over one year) obtained constraining the maximum distance from the Earth to $d_{\max }$. Initial mass is $m_{0}=1000 \mathrm{~kg}$.

\begin{tabular}{ccccccc}
\hline \hline$\beta_{0}$ & $d_{\max }, \mathrm{AU}$ & $\min _{t} d(t), \mathrm{AU}$ & $\max _{t} d(t), \mathrm{AU}$ & $m_{f}, \mathrm{~kg}$ & $m_{\text {prop }} / m_{0}$ & $\max T, \mathrm{~N}$ \\
\hline \multirow{6}{*}{0.0} & 0.018 & 0.015616 & 0.018 & 842.666099 & 0.157334 & 0.174290 \\
& 0.017 & 0.015583 & 0.017 & 841.886048 & 0.158114 & 0.170876 \\
& 0.016 & 0.015466 & 0.016 & 840.580282 & 0.159420 & 0.166309 \\
& 0.015 & 0.014988 & 0.015 & 838.280770 & 0.161719 & 0.162189 \\
& 0.014 & 0.013999 & 0.014 & 834.122246 & 0.165878 & 0.167615 \\
& 0.013 & 0.013000 & 0.013 & 827.345636 & 0.172654 & 0.175659 \\
& 0.012 & 0.011999 & 0.012 & 817.098782 & 0.182901 & 0.187183 \\
\hline \multirow{6}{*}{0.05} & 0.018 & 0.013179 & 0.018 & 898.507259 & 0.101493 & 0.142394 \\
& 0.017 & 0.013196 & 0.017 & 896.688888 & 0.103311 & 0.138714 \\
& 0.016 & 0.013201 & 0.016 & 894.203213 & 0.105797 & 0.137004 \\
& 0.015 & 0.013183 & 0.015 & 890.818185 & 0.109182 & 0.134783 \\
& 0.014 & 0.013105 & 0.014 & 886.186387 & 0.113814 & 0.132355 \\
& 0.013 & 0.012838 & 0.013 & 879.749327 & 0.120251 & 0.129667 \\
& 0.012 & 0.012000 & 0.012 & 870.546037 & 0.129454 & 0.133296 \\
\hline \multirow{6}{*}{0.1} & 0.018 & 0.012012 & 0.018 & 916.731609 & 0.083268 & 0.126068 \\
& 0.017 & 0.012025 & 0.017 & 914.433408 & 0.085567 & 0.124981 \\
& 0.016 & 0.012036 & 0.016 & 911.558363 & 0.088442 & 0.122102 \\
& 0.015 & 0.012037 & 0.015 & 907.916377 & 0.092084 & 0.120447 \\
& 0.014 & 0.012020 & 0.014 & 903.226895 & 0.096773 & 0.119052 \\
\hline \hline & 0.013 & 0.011952 & 0.013 & 897.070392 & 0.102930 & 0.117095 \\
& 0.012 & 0.011749 & 0.012 & 888.767765 & 0.111232 & 0.114955 \\
\hline \hline
\end{tabular}

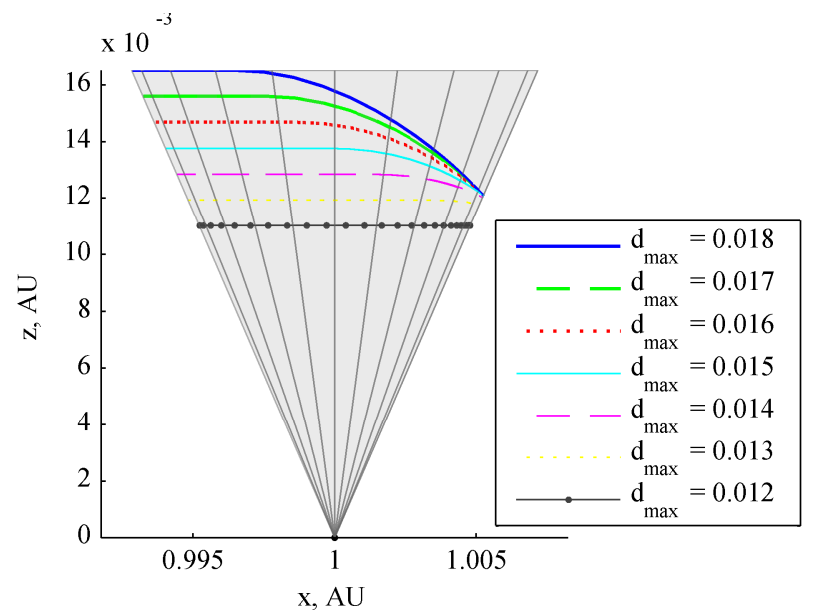

Fig. 26. A family of optimal orbits, constrained to several values of $d_{\max }$, for $\beta_{0}=0.05$. 
Another possible requirement for the pole-sitter spacecraft payload could be to maintain a constant or quasiconstant distance from the Earth. This can be useful for a camera with a fixed focal length, in which the field of view cannot be varied, and thus a constant distance from the Earth is desirable such that the instrument can be designed for that distance. It was shown already that if the orbit is flat, then the shape-based approach can be used to compute the optimal controls, and minimize the propellant mass. In this section, however, a trade-off between the flatness of the orbit and the additional propellant mass required will be shown. Furthermore, it is assumed that there is no particular requirement on the maximum distance $d_{\max }$. To achieve this, in the cost function a weighed penalty that considers the movement of the spacecraft along $\hat{\mathbf{z}}$ is introduced. If it is noted that a flat orbit is the one in which the velocity along $\hat{\mathbf{z}}$ is null during the whole orbit, a possible choice of the cost function could be:

$$
J=-m_{f}+\frac{w}{2 \pi} \int_{0}^{2 \pi} v_{z}^{2} \mathrm{~d} t
$$

By varying the weight $w$, a trade-off of propellant mass against a low average of the spacecraft velocity along $\hat{\mathbf{z}}$ is possible. Note that the case $w=0$ coincides with the optimization of the propellant mass only. Table 3 shows a summary of the characteristics of the optimal orbits found using the cost function in Eq. (39), for the three different values of $\beta_{0}$ and for several values of the weight $w$. These values have been determined by trial and error, and considering the relative order of magnitude of the two addends in the cost function (in non-dimensional units). As expected, the orbits flatten at the cost of some additional propellant mass. Note that the additional propellant mass needed to flatten the orbit, in the case of pure SEP, is only of order $2 \mathrm{~kg}$; instead, the cost of flattening the orbit increases when a hybrid sail is considered: for example, in the case $\beta_{0}=0.1$, a flat orbit costs about $16 \mathrm{~kg}$ more than the optimal orbit. However, a flat orbit at optimal distance with $\beta_{0}=0.1$ is cheaper to maintain than the optimal orbit with $\beta_{0}=0.05$. For values of $w$ higher that those presented in the table, the orbit is substantially flat, stabilizing to a distance of $0.017,0.0175,0.0177 \mathrm{AU}$ for $\beta_{0}=0,0.05,0.1$ respectively. These values represent the optimal distance for a flat pole-sitter orbit, as a function of $\beta_{0}$, and coincide with the minimum of the curves plotted in Fig. 13.

Table 3. Summary of optimal orbits obtained minimizing a weighed sum of propellant mass and velocity in $\mathrm{z}$. Different values of the weight $w$ are considered. Initial mass is $m_{0}=1000 \mathrm{~kg}$.

\begin{tabular}{ccccccc}
\hline \hline$\beta_{0}$ & $w$ & $\min _{t} d(t), \mathrm{AU}$ & $\max _{t} d(t), \mathrm{AU}$ & $m_{f}, \mathrm{~kg}$ & $m_{\text {prop }} / m_{0}$ & $\max T, \mathrm{~N}$ \\
\hline \multirow{4}{*}{0.0} & 0 & 0.015675 & 0.020333 & 843.430417 & 0.156570 & 0.180668 \\
& $5 \cdot 10^{-15}$ & 0.016516 & 0.017801 & 842.199379 & 0.157801 & 0.166281 \\
& $5 \cdot 10^{-14}$ & 0.016917 & 0.017099 & 841.264629 & 0.158735 & 0.176469 \\
& $1 \cdot 10^{-13}$ & 0.016953 & 0.017047 & 841.174169 & 0.158826 & 0.158635 \\
& $2 \cdot 10^{-13}$ & 0.016975 & 0.017022 & 841.125662 & 0.158874 & 0.160165 \\
\hline \multirow{4}{*}{0.05} & 0 & 0.013116 & 0.023416 & 901.894908 & 0.098105 & 0.141267 \\
& $5 \cdot 10^{-15}$ & 0.014744 & 0.019960 & 899.638660 & 0.100361 & 0.134823 \\
& $5 \cdot 10^{-14}$ & 0.016982 & 0.017942 & 894.916402 & 0.105084 & 0.134742 \\
& $1 \cdot 10^{-13}$ & 0.017262 & 0.017763 & 894.270074 & 0.105730 & 0.135284 \\
& $2 \cdot 10^{-13}$ & 0.017411 & 0.017668 & 893.914791 & 0.106085 & 0.135428 \\
\hline \multirow{4}{*}{0.1} & 0 & 0.011900 & 0.028253 & 925.148293 & 0.074852 & 0.134605 \\
& $5 \cdot 10^{-15}$ & 0.013981 & 0.021394 & 919.943583 & 0.080056 & 0.122209 \\
& $5 \cdot 10^{-14}$ & 0.017031 & 0.018251 & 911.326314 & 0.088674 & 0.126967 \\
& $1 \cdot 10^{-13}$ & 0.017393 & 0.018022 & 910.306349 & 0.089694 & 0.128146 \\
& $2 \cdot 10^{-13}$ & 0.017588 & 0.017907 & 909.759657 & 0.090240 & 0.129009 \\
\hline
\end{tabular}




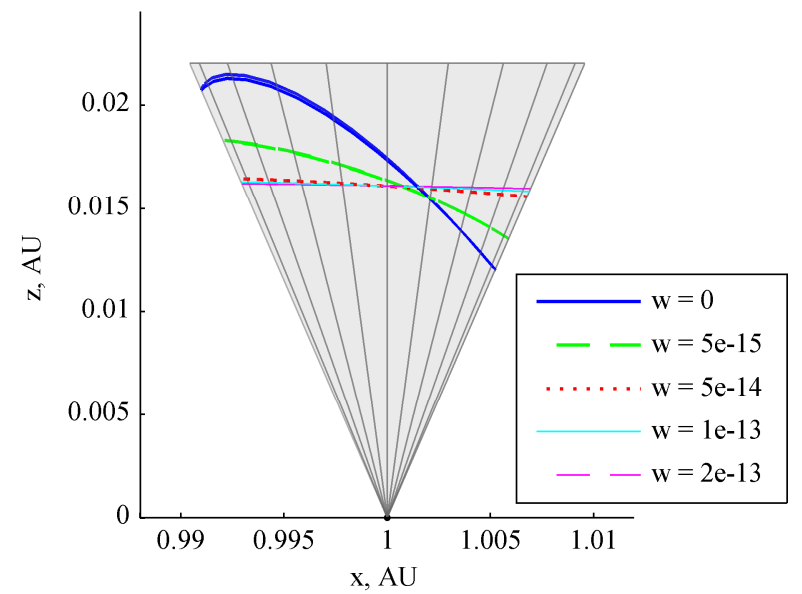

Fig. 27. A family of optimal orbits, for different values of the cost function weight $w$, and $\beta_{0}=0.05$.

\section{Mass Budget}

So far, this paper has presented the performance of several different optimal pole-sitter orbits, showing the advantages in terms of propellant mass (or propellant mass fraction) of the hybrid spacecraft with respect to the pure SEP system. However, the hybrid spacecraft is a more complex system, mainly due to the presence of the solar sail and the need to have a steerable SEP thrust vector with respect to the sail, which is assumed to be fixed with the main spacecraft bus. Due to the additional subsystem mass, it is interesting to assess whether the hybrid system allows a greater payload mass $m_{p l}$, with the same initial spacecraft mass $m_{0}$. A full analysis of the system mass budget is beyond the scopes of this paper; however a few design points will be presented, to show the feasibility and applicability of the hybrid spacecraft.

For sake of comparison, the technological assumptions are based on what was chosen in Ref. 17. In that work, the authors computed the requirements for a spacecraft to be stationary in the Sun-Earth rotation frame, placed at $0.01831 \mathrm{AU}$ above the North Pole at the summer solstice (hence above the Lagrange point $L_{1}$ ), a solar sail with $\beta_{0}=0.03$, a payload mass of $100 \mathrm{~kg}$, a SEP specific impulse of $3200 \mathrm{~s}$, and a 5 year mission timeframe. The same specific impulse is used in this section.

For a preliminary mass budget, the total spacecraft mass can be split as:

$$
m_{0}=m_{\text {prop }}+m_{\text {tank }}+n_{\text {thrusters }}\left(m_{S E P}+m_{\text {gimbal }}\right)+m_{s}+m_{T F}+m_{p l}
$$

where $m_{\text {prop }}$ is the propellant mass necessary for a given mission duration $t_{\text {mission }}$, and is computed solving the optimal control problem described before for an estimated initial mass $m_{0, \text { guess }}$. The orbit is optimized for one period, and the same orbit is used throughout the whole mission, for the remaining periods. The mass of the tanks is $m_{\text {tank }}=0.1 m_{\text {prop }},{ }^{40}$ the number of thrusters is $n_{\text {thrusters }}=2$, for redundancy and such that a second thruster can be used after the performance of the first has degraded; the mass of the engine is $m_{S E P}=k_{S E P} P_{S E P, \text { max }}$, with $k_{S E P}=20 \mathrm{~kg} / \mathrm{kW}$ (NASA solar electric propulsion technology application readiness class engine). ${ }^{30}$ The maximum power $P_{S E P, \max }$ required by the SEP subsystem is computed as a function of the maximum thrust $T_{\max }$ required during the mission, in the following way: $P_{S E P, \max }=T_{\max } v_{e} / 2 \eta_{S E P}$, where $\eta_{S E P}=0.7$ is the efficiency of converting electrical energy. ${ }^{41}$ The upper limit on the SEP thrust $T_{u}$ is not considered here, as it is assumed that the size and performance of the engine scales up together with the mass of the spacecraft. For the hybrid spacecraft, $m_{\text {gimbal }}=0.3 m_{S E P},{ }^{40}$ for the pure $\mathrm{SEP}$, instead, $m_{\text {gimbal }}=0$. The total sail area (highly reflective surface + TFSC) can be computed starting from the assumed values of $\beta_{0}$ and $m_{0}$, from Eq. (9), as $A=\beta_{0} m_{0} / \sigma^{*}$. The mass of the thin film solar cells is proportional to their area: $m_{T F}=\sigma_{T F} A_{T F}$, where $\sigma_{T F S C}=100 \mathrm{~g} / \mathrm{m}^{2}$ (Ref. 16). The area was conservatively assumed to be $5 \%$ of the total area of the sail. ${ }^{17}$ Here, a more precise value can be estimated, as a function of the maximum power. In particular, for the pure SEP spacecraft, the solar panels are usually kept perpendicular to the Sun vector, and therefore the area of TSFC necessary to guarantee the required power is $A_{T F}=P_{S E P, \max } / W \eta_{T F}$, with $\eta_{T F}=0.05$ due 
to the relatively low efficiency of the thin film, and energy flux density of the Sun $W=1367 \mathrm{~W} / \mathrm{m}^{2}$, considered constant at $1 \mathrm{AU}$. In the hybrid spacecraft, instead, the TFSC is part of the reflective surface, and therefore their pitch with respect to the Sun vector is given by the clock angle of the sail $\alpha=\alpha_{T_{\max }}$ at the instant when the maximum thrust is required. Consequently, in the hybrid case, the area of the TFSC shall be augmented according to $A_{T F}=P_{S E P, \max } / W \eta_{T F} \cos \alpha_{T_{\max }}$. The area of the sail is simply $A_{s}=A-A_{T F}$, and its mass is $m_{s}=\sigma_{s} A_{s} . \sigma_{s}$, the mass per unit area of the sail, or sail loading, is a critical parameter that depends on the solar sail technology. Currently, realistic values are on the order of $25-30 \mathrm{~g} / \mathrm{m}^{2}$ (Ref. 18), but near-term technological developments should allow values of $10 \mathrm{~g} / \mathrm{m}^{2}{ }^{42}$. Ultra-thin (around $2 \mu \mathrm{m}$ of thickness) sails are expected in the mid- to long-term timeframe ${ }^{43}$ : they can lead, for large sails, to loadings of the order of $5 \mathrm{~g} / \mathrm{m}^{2}$.

Eq. (41) can be solved, for example for $m_{0}$, having assigned a payload mass, with an iterative method. Table 4 presents two pole-sitter mission scenarios: a short mission (5 years), and a long mission ( 8 years). In both the cases, the payload mass to be carried is $m_{p l}=100 \mathrm{~kg}$, to allow comparison with previous work. ${ }^{17}$ Three different spacecraft were considered, for each scenario: a pure SEP, a near-term hybrid sail (sail loading of $7.5 \mathrm{~g} / \mathrm{m}^{2}$ ) and a mid- to long-term hybrid sail $\left(5 \mathrm{~g} / \mathrm{m}^{2}\right)$. The value of $\beta_{0}$ was consequently selected for each case, to minimize the initial mass.

For 5 years, the pure SEP spacecraft weighs about $434 \mathrm{~kg}$ at launch: this value is comparable to the static equilibrium case above $L_{1}$ that was presented in Ref. 17. For the 5-year mission, the mid-term sail is not convenient, as the launch mass would be slightly higher $(459 \mathrm{~kg})$. A major improvement is found considering a lower sail loading, as in the long-term spacecraft: in this case, the initial mass drops to $384 \mathrm{~kg}$.

It is important to underline here that the saving in initial mass is not as important as what was found for the static equilibrium case, in which the initial mass for the hybrid spacecraft (with a sail loading of $10 \mathrm{~g} / \mathrm{m}^{2}$ ) dropped to 288 $\mathrm{kg} .{ }^{17}$ This is due to the fact that at the summer solstice the total acceleration to be counterbalanced by the SEP and sail is minimum, with respect to that in other positions of the polar cone (see for example Fig. 17, in which the position of the static equilibrium spacecraft is at $t=182.5 \mathrm{~d}$ ). Therefore, at that point, the contribution of the sail is maximized, and staying stationary in this position exploits this advantage during the whole mission. It can be deduced that following the North Pole of the Earth results in a more expensive trajectory, in terms of propellant mass, with respect to a stationary spacecraft in an equivalent equilibrium position above the $L_{1}$ Lagrange point.

However, the improvement of the hybrid spacecraft with respect to the pure SEP spacecraft becomes important considering the longer 8-year mission. In this case, for pure SEP, a $2418 \mathrm{~kg}$ spacecraft and a maximum thrust of $0.42 \mathrm{~N}$ is required. Instead, adding a mid-term sail brings these values down to $1924 \mathrm{~kg}$ and $0.3 \mathrm{~N}$. As expected, further improvements are obtained for the long-term spacecraft.

Table 4. Mass budget for different mission durations and values of sail lightness number and sail loading for $m_{p l}=100 \mathrm{~kg}$.

\begin{tabular}{lcccccc}
\hline \hline Spacecraft & Pure SEP & $\begin{array}{c}\text { Mid-term } \\
\text { hybrid }\end{array}$ & $\begin{array}{c}\text { Long-term } \\
\text { hybrid }\end{array}$ & Pure SEP & $\begin{array}{c}\text { Mid-term } \\
\text { hybrid }\end{array}$ & $\begin{array}{c}\text { Long-term } \\
\text { hybrid }\end{array}$ \\
\hline$t_{\text {mission }}, \mathrm{y}$ & 5 & 5 & 5 & 8 & 8 & 8 \\
$\sigma_{s}, \mathrm{~g} / \mathrm{m}^{2}$ & - & 7.5 & 5 & - & 7.5 & 5 \\
$\beta_{0}$ & 0 & 0.02 & 0.03 & 0 & 0.02 & 0.04 \\
\hline$m_{0}, \mathrm{~kg}$ & 434.6450 & 459.0042 & 384.2920 & 2418.7 & 1923.9 & 971.4498 \\
$m_{p l}, \mathrm{~kg}$ & 100 & 100 & 100 & 100 & 100 & 100 \\
$m_{p r o p}, \mathrm{~kg}$ & 239.8382 & 206.8880 & 161.1140 & 1749.6 & 1157.3 & 522.8407 \\
$m_{s}, \mathrm{~kg}$ & - & 44.7396 & 37.5298 & - & 187.5263 & 126.6162 \\
$m_{T F S C}, \mathrm{~kg}$ & 2.4990 & 3.4770 & 2.9178 & 13.9068 & 14.5739 & 7.4148 \\
$A_{s}, \mathrm{~m}^{2}$ & - & 5965.3 & 7505.9 & - & 25003 & 25323 \\
$A_{T F S C}, \mathrm{~m}^{2}$ & 24.9905 & 34.7702 & 29.1776 & 139.0679 & 145.7395 & 74.1482 \\
$T_{\text {max }}, \mathrm{N}$ & 0.0762 & 0.0714 & 0.0571 & 0.4239 & 0.2991 & 0.1392 \\
\hline \hline
\end{tabular}


Furthermore, it was found that the pure SEP spacecraft option is not feasible if the mission time is longer than about 8.8 years, and therefore for longer missions, the hybrid spacecraft is the only option. For example, the longterm hybrid can carry the same payload for 10 years with an initial mass of about $4000 \mathrm{~kg}$.

It is interesting to note that, despite the benefit of the solar sail to the pole-sitter spacecraft, it is not as good as the benefit that the static equilibrium spacecraft above $L_{1}$ receives. ${ }^{17}$ However, it was shown that the hybrid spacecraft starts to be beneficial over the pure SEP for $\sigma_{s} \leq 7.5 \mathrm{~g} / \mathrm{m}^{2}$. As a comparison, it was found ${ }^{18}$ that, in the case of direct interplanetary transfers, the hybrid propulsion decreased the payload mass fraction, with respect to pure SEP, only if $\sigma_{s} \leq 2 \mathrm{~g} / \mathrm{m}^{2}$. Therefore, it can be concluded that the hybrid sail is more beneficial for the polesitter problem, and it is expected that near- to mid-term technology will be able to provide sails with loadings that can enable the hybrid pole-sitter mission.

\section{Conclusions}

Optimal pole-sitter orbits were presented using hybrid solar electric propulsion (SEP) and solar sail propulsion. The combination of the two propulsion systems allows propellant saving over the pure SEP spacecraft, enabling longer missions; at the same time, the presence of low-thrust propulsion enables orbits that cannot be achieved with a pure sail. The analysis took into account constant-distance flat orbits, as well as solutions in which the spacecraft varies its altitude above either pole. For flat orbits, a shape based approach was used to find optimal solutions. However, if the distance from Earth is not pre-determined, optimal orbits were found solving an optimal control problem. A direct method based on pseudo-spectral discretization was demonstrated to be reliable and suitable for this aim, guaranteeing fast convergence to the optimal solution. Optimal, variable-distance orbits resulted in additional propellant mass saving. Furthermore, intermediate orbits were investigated, trading off either the maximum allowed distance from the Earth, or a requirement on the variation of the Earth polar distance.

A preliminary mass budget showed that mid-term sail technology allows lower launch mass for long duration pole-sitter missions (6 years and above), and enables even longer missions, that are unfeasible with a pure SEP spacecraft.

\section{Acknowledgements}

This work was funded by the European Research Council, as part of project 227571 VISIONSPACE. The authors thank Dr. Victor M. Becerra, of the School of Systems Engineering, University of Reading, Reading, UK for providing the software PSOPT freely, as well as advices on its use.

\section{References}

${ }^{1}$ Wallace, N. C., "Testing of the Qinetiq T6 Thruster in Support of the Esa Bepicolombo Mercury Mission," Proceedings of the Space Propulsion 2004 - 4th International Spacecraft Propulsion Conference, June 2, 2004 June 4, 2004, European Space Agency, Sardinia, Italy, 2004, pp. 479-484.

${ }^{2}$ McInnes, C. R., Solar Sailing: Technology, Dynamics and Mission Applications, Springer-Praxis Books in Astronautical Engineering, Springer-Verlag, Berlin, 1999.

${ }^{3}$ Tsander, F. A., From a Scientific Heritage (Translation Of "Iz Nauchnogo Naslediya"), Nasa Technical Translation, NASA, 1969.

${ }^{4}$ Baoyin, H., and McInnes, C. R., "Solar Sail Equilibria in the Elliptical Restricted Three-Body Problem," Journal of Guidance, Control, and Dynamics, Vol. 29, No. 3, 2006, pp. 538-543.

${ }^{5}$ Mengali, G., and Quarta, A. A., "Solar Sail Trajectories with Piecewise-Constant Steering Laws," Aerospace Science and Technology, Vol. 13, No. 8, 2009, pp. 431-441. doi: 10.1016/j.ast.2009.06.007

${ }^{6}$ Kawaguchi, J. i., Mimasu, Y., Mori, O., Funase, R., Yamamoto, T., et al., "Ikaros - Ready for Lift-Off as the World's First Solar Sail Demonstration in Interplanetary Space," Proceedings of the $60^{\text {th }}$ International Astronautical Congress, IAC 2009, Daejeon, Korea, 2009.

${ }^{7}$ Mengali, G., and Quarta, A. A., "Optimal Three-Dimensional Interplanetary Rendezvous Using Nonideal Solar Sail," Journal of Guidance, Control, and Dynamics, Vol. 28, No. 1, 2005, pp. 173-177.

${ }^{8}$ Baoyin, H., and McInnes, C. R., "Solar Sail Orbits at Artificial Sun-Earth Libration Points," Journal of Guidance, Control, and Dynamics, Vol. 28, No. 6, 2005, pp. 1328-1331.

${ }^{9}$ Simo, J., and McInnes, C. R., "Solar Sail Orbits at the Earth-Moon Libration Points," Communications in Nonlinear Science and Numerical Simulation, Vol. 14, No. 12, 2009, pp. 4191-4196. doi: 10.1016/j.cnsns.2009.03.032 
${ }^{10}$ McInnes, C. R., “Artificial Lagrange Points for a Partially Reflecting Flat Solar Sail,” Journal of Guidance, Control, and Dynamics, Vol. 22, No. 1, 1999, pp. 185-187.

${ }^{11}$ McInnes, C. R., "Dynamics, Stability, and Control of Displaced Non-Keplerian Orbits," Journal of Guidance, Control, and Dynamics, Vol. 21, No. 5, 1998, pp. 799-805.

${ }^{12}$ Baig, S., and McInnes, C. R., "Light-Levitated Geostationary Cylindrical Orbits Are Feasible," Journal of Guidance, Control, and Dynamics, Vol. 33, No. 3, 2010, pp. 782-793. doi: 10.2514/1.46681

${ }^{13}$ Waters, T. J., and McInnes, C. R., "Periodic Orbits above the Ecliptic in the Solar-Sail Restricted Three-Body Problem," Journal of Guidance, Control, and Dynamics, Vol. 30, No. 3, 2007, pp. 687-693. doi: 10.2514/1.26232

${ }^{14}$ Biggs, J. D., McInnes, C. R., and Waters, T., "Control of Solar Sail Periodic Orbits in the Elliptic Three-Body Problem," Journal of Guidance, Control, and Dynamics, Vol. 32, No. 1, 2009, pp. 318-320. doi: 10.2514/1.38362

${ }^{15}$ Waters, T. J., and McInnes, C. R., "Invariant Manifolds and Orbit Control in the Solar Sail Three-Body Problem," Journal of Guidance, Control, and Dynamics, Vol. 31, No. 3, 2008, pp. 554-562. doi: 10.2514/1.32292

${ }^{16}$ Leipold, M., and Götz, M., "Hybrid Photonic/Electric Propulsion," Kayser-Threde GmbH, 2002.

${ }^{17}$ Baig, S., and McInnes, C. R., "Artificial Three-Body Equilibria for Hybrid Low-Thrust Propulsion," Journal of Guidance, Control, and Dynamics, Vol. 31, No. 6, 2008, pp. 1644-1655. doi: 10.2514/1.36125

${ }^{18}$ Mengali, G., and Quarta, A. A., "Trajectory Design with Hybrid Low-Thrust Propulsion System," Journal of Guidance, Control, and Dynamics, Vol. 30, No. 2, 2007, pp. 419-426. doi: 10.2514/1.22433

${ }^{19}$ Mengali, G., and Quarta, A. A., "Tradeoff Performance of Hybrid Low-Thrust Propulsion System," Journal of Spacecraft and Rockets, Vol. 44, No. Compendex, 2007, pp. 1263-1270. doi: 10.2514/1.30298

${ }^{20}$ Quarta, A. A., Mengali, G., and Janhunen, P., "Optimal Interplanetary Rendezvous Combining Electric Sail and High Thrust Propulsion System," Acta Astronautica, Vol. In Press, Corrected Proof, 2010. doi: 10.1016/j.actaastro.2010.01.024

${ }^{21}$ Simo, J., and McInnes, C. R., "Displaced Periodic Orbits with Low-Thrust Propulsion," Proceedings of the $19^{\text {th }}$ AAS/AIAA Space Flight Mechanics Meeting, Savannah, Georgia, USA, 2009.

${ }^{22}$ Mori, O., Sawada, H., Funase, R., Endo, T., Morimoto, M., et al., "Development of First Solar Power Sail Demonstrator - Ikaros," Proceedings of the 21st International Symposium on Space Flight Dynamics, ISSFD 2009, CNES, Toulouse, France, 2009.

${ }^{23}$ Macdonald, M., "Solar Sail Mission Applications and Future Advancement," Proceedings of the $2^{\text {nd }}$ International Symposium on Solar Sailing (ISSS 2010), New York, USA, 2010.

${ }^{24}$ Driver, J. M., "Analysis of an Arctic Polesitter," Journal of Spacecraft and Rockets, Vol. 17, No. 3, 1980. doi: $10.2514 / 3.57736$

${ }^{25}$ McInnes, C. R., and Mulligan, P., "Final Report: Telecommunications and Earth Observations Applications for Polar Stationary Solar Sails," National Oceanographic and Atmospheric Administration (NOAA)/University of Glasgow, Department of Aerospace Engineering, 2003.

${ }^{26}$ Forward, R. L., "Statite: A Spacecraft That Does Not Orbit," Journal of Spacecraft and Rockets, Vol. 28, No. 5, 1991, pp. 606-611. doi: 10.2514/3.26287

${ }^{27}$ Wertz, J. R., and Larson, W. J. (eds.), Space Mission Analysis and Design, Third Edition, Space Technology Library, Microcosm press/Kluwer Academic Publishers, El Segundo, California, USA, 1999.

${ }^{28}$ "Cryosat Mission and Data 2007, http://esamultimedia.esa.int/docs/Cryosat/Mission_and_Data_Descrip.pdf [cited 2 January 2007].

${ }^{29}$ Dachwald, B., Mengali, G., Quarta, A. A., and Macdonald, M., "Parametric Model and Optimal Control of Solar Sails with Optical Degradation," Journal of Guidance, Control, and Dynamics, Vol. 29, No. 5, 2006, pp. 1170-1178. doi: 10.2514/1.20313

${ }^{30}$ Brophy, J., "Advanced Ion Propulsion Systems for Affordable Deep-Space Missions," Acta Astronautica, Vol. 52, 2003, pp. 309-316. doi: 10.1016/S0094-5765(02)00170-4

${ }^{31}$ Petropoulos, A. E., and Longuski, J. M., "Shape-Based Algorithm for Automated Design of Low-Thrust, Gravity-Assist Trajectories," Journal of Spacecraft and Rockets, Vol. 41, No. 5, 2004, pp. 787-796.

${ }^{32}$ Powell, M. J. D., "A Fast Algorithm for Nonlinearly Constrained Optimization Calculations," Numerical Analysis, edited by G.A. Watson, Lecture Notes in Mathematics, Springer, Berlin, 1978, pp. 144-157.

${ }^{33}$ Fornberg, B., A Practical Guide to Pseudospectral Methods, Cambridge Monographs on Applied and Computational Mathematics, Cambridge University Press, Cambridge, NY, USA, 1996.

${ }^{34}$ Fahroo, F., and Ross, I. M., "Direct Trajectory Optimization by a Chebyshev Pseudospectral Method," Journal of Guidance, Control, and Dynamics, Vol. 25, No. Compendex, 2002, pp. 160-166. doi: 10.2514/2.4862 
${ }^{35}$ Elnagar, G., Kazemi, M. A., and Razzaghi, M., "Pseudospectral Legendre Method for Discretizing Optimal Control Problems," IEEE Transactions on Automatic Control, Vol. 40, No. 10, 1995, pp. 1793-1796. doi: $10.1109 / 9.467672$

${ }^{36}$ Becerra, V. M., "Psopt Optimal Control Solver User Manual,” 2009, http://code.google.com/p/psopt [cited 14 May 2009].

${ }^{37}$ Wächter, A., and Biegler, L. T., "On the Implementation of a Primal-Dual Interior Point Filter Line Search Algorithm for Large-Scale Nonlinear Programming," Mathematical Programming, Vol. 106, No. 1, 2006, pp. 2557.

${ }^{38}$ Betts, J. T., Practical Methods for Optimal Control Using Nonlinear Programming, Advances in Design and Control, Society for Industrial \& Applied Mathematics, 2001.

${ }^{39}$ Lewis, F. L., and Syrmos, V. L., Optimal Control, Second Edition, John Wiley \& Sons, Inc., New York, 1995.

${ }^{40}$ Gershman, R., and Seybold, C., "Propulsion Trades for Space Science Missions," Acta Astronautica, Vol. 45, No. 4-9, 1999, pp. 541-548. doi: 10.1016/S0094-5765(99)00174-5

${ }^{41}$ Kitamura, S., Ohkawa, Y., Hayakawa, Y., Yoshida, H., and Miyazaki, K., "Overview and Research Status of the Jaxa 150-Mn Ion Engine," Acta Astronautica, Vol. 61, No. 1-6, 2007, pp. 360-366. doi: 10.1016/j.actaastro.2007.01.010

${ }^{42}$ Dachwald, B., "Solar Sail Performance Requirements for Missions to the Outer Solar System and Beyond," Proceedings of the 55 $5^{\text {th }}$ International Astronautical Congress (IAC 2004), Vol. 11, International Astronautical Federation, IAF, Vancouver, Canada, 2004, pp. 7345-7353.

${ }^{43}$ Murphy, D. M., Murphey, T. W., and Gierow, P. A., "Scalable Solar-Sail Subsystem Design Considerations," Proceedings of the $43^{\text {rd }}$ Structures, Structural Dynamics, and Materials Conference, American Inst. Aeronautics and Astronautics Inc., Denver, Colorado, 2002. 